\title{
Burst Firing of the Ventral Tegmental Area Neurons through Bicuculline-Induced GABA-A Receptor Blockade
}

\author{
Fereshteh Tale ${ }^{1}$, Firouz Ghaderi Pakdel ${ }^{1,2^{*}}$, Parviz Shahabi ${ }^{3}$, Somayyeh Naderi ${ }^{4}$ \\ ${ }^{1}$ Department of Physiology, Faculty of Medicine, Urmia University of Medical Sciences, Urmia, Iran \\ ${ }^{2}$ Neurophysiology Research Center, Faculty of Medicine, Urmia University of Medical Sciences, Urmia, Iran \\ ${ }^{3}$ Neuroscience Research Center, Tabriz University of Medical Sciences, Tabriz, Iran \\ ${ }^{4}$ Danesh Pey Hadi Co, Urmia Health Technology Development Center, Research and Technology Vice Chancellor, Urmia University of \\ Medical Sciences, Urmia, Iran
}

\section{ABSTRACT}

Introduction: Ventral Tegmental Area (VTA), as a major source of dopaminergic (DA) neurons, has crucial roles in the vital and pathologic conditions, such as drug dependence and depression. The receptors on the VTA-DA neurons with different density and diversity have modulatory effects on their target tissues, such as the cerebral cortex. The aim of the present study was to study the effect of GABA-A receptor blockade on the VTA-DA neuronal firing pattern. Materials and Methods: Male Wistar rats ( $200 \mathrm{gr})$ were randomly allocated in 8 groups with equal substitutive numbers (control, sham, and treatments). The VTA-DA neuronal firing patterns (simple-tonic or burst firing) were acquisitioned under urethane anesthesia and stereotaxic approach. Bicuculline as a GABA-A receptor antagonist were infused microiontophoretically intracerebroventricularly (5, $25,50,500,1000$, and 2500 ngr) and peri-injection neuronal firing were captured for burst firing detection. Results: The simple-tonic spiking of the VTA-DA neurons in the pre-injection period was about 5.78 spikes/sec overall with stable firing. Bicuculline microinjection dose-dependently modulated burst firing pattern. The abovementioned amounts of Bicuculline induced 5.2, 10.4, $23.4,28.8,35.9,36.17$ percent of neurons to fire burst with duration of 5.2, 8.7, 24.45, 29.87, 40.56, and 50.34 minutes, respectively. The post injection simple-tonic firing rates were also dose-dependently elevated from 8 to 358 percent of the pre-injection levels. Conclusion: The results showed that GABA-A receptors on the VTA-DA neurons can have regulatory roles on their firing patterns. The external GABAergic afferents or internal interneurons that mediate GABA modulation on the VTA-DA neurons can interfere in the efferent functions of the VTA-DA neurons.

\section{Key words:}

1. Ventral Tegmental Area 2. Dopaminergic Neurons 3. Neurons

*Corresponding Author: Firouz Ghaderi Pakdel

E-mail: info@fgpakdel.com 


\title{
شليك انفجارى نورونهاى ناحيةٌ تخمنتوم شكمى از طريق مهار كيرنده GABA-A القاء شده با بيكوكولين
}

\author{
فرشته طالع'، فيروز قادرى ياكدل ‘،"، يرويز شهابى"، سميه نادرى \\ 'كروه فيزيولوزى، دانشكده بزشكى، دانشكاه علوم يزشكى اروميه، اروميه، ايران

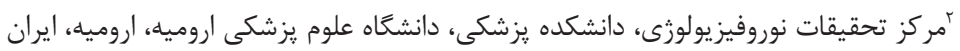

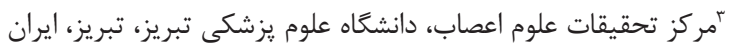

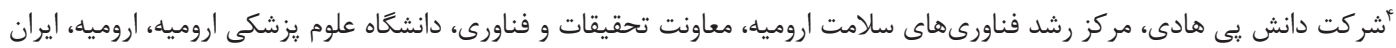

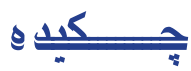

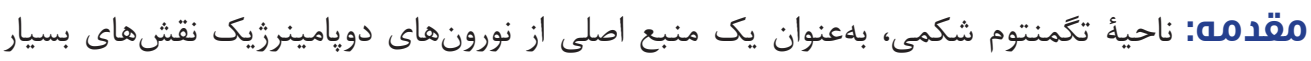

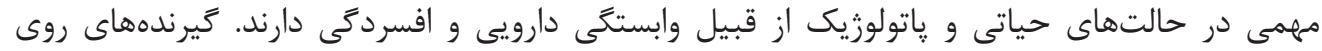

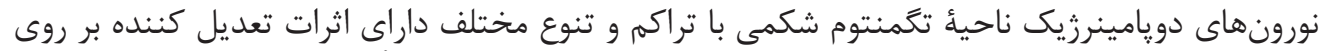

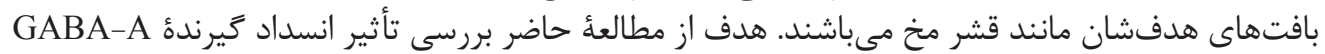

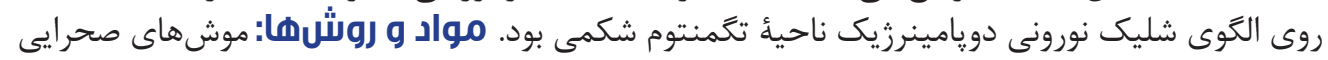

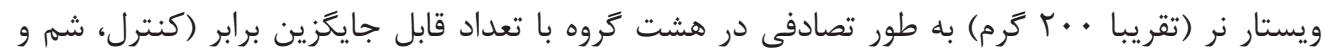

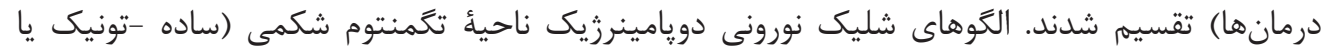

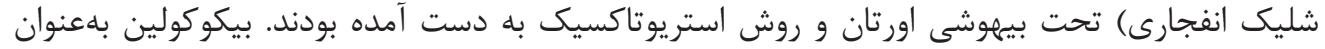

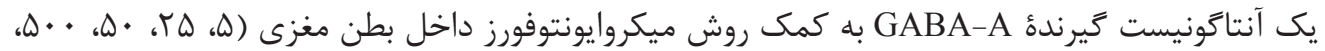

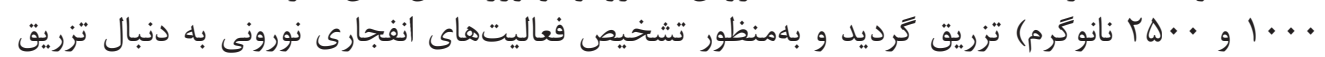

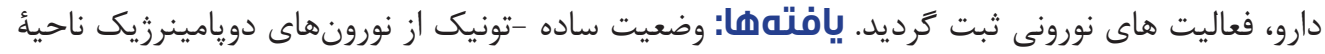

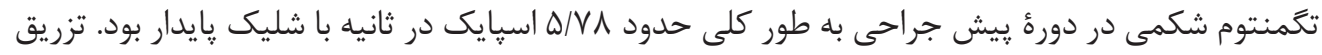

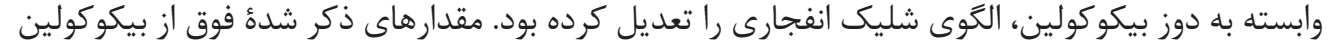

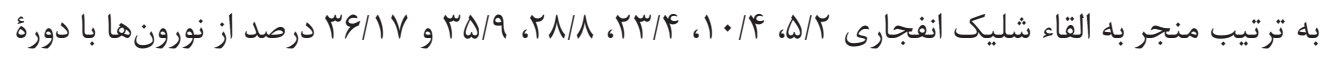

كليد وازهها:

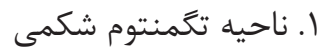

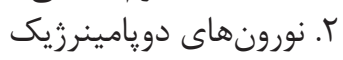

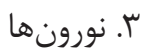

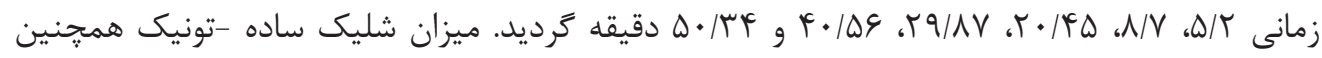

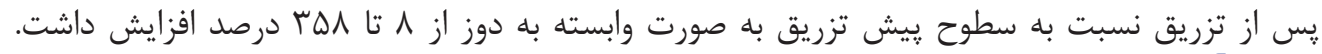

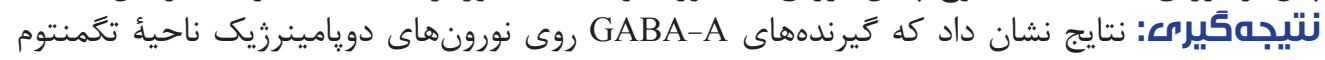

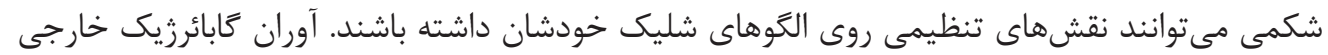

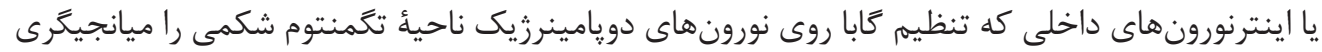

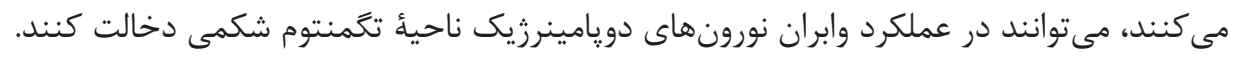

"نويسنده مسئول: فيروز قادرى پاكدل info@fgpakdel.com آدرس الكترونيكى باكنى فيرون 


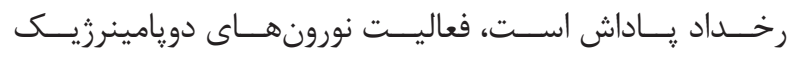

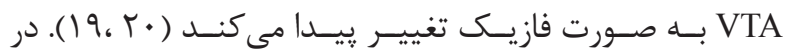

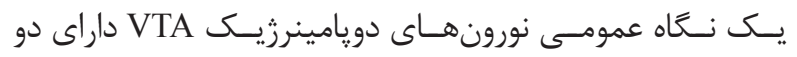

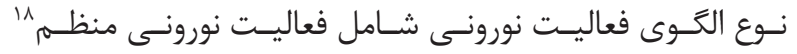

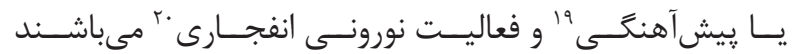

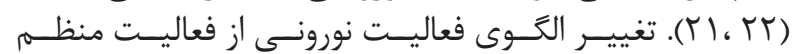

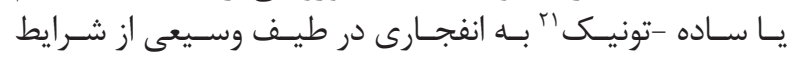

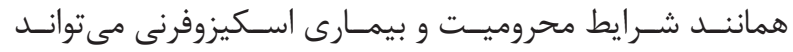

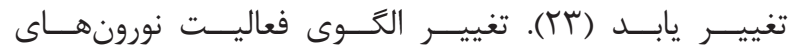

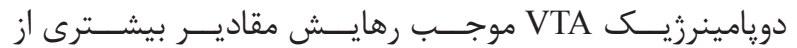

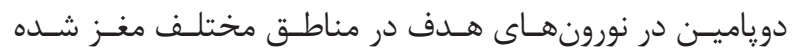

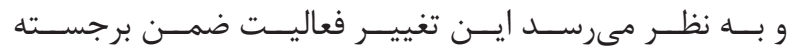

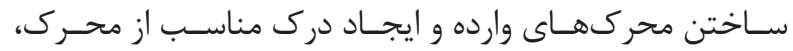

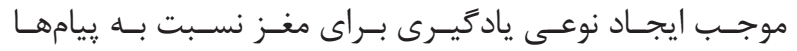

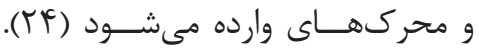

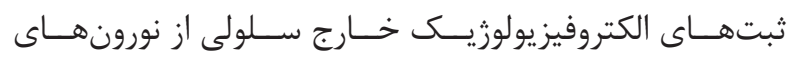

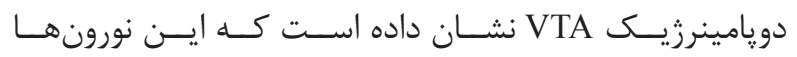

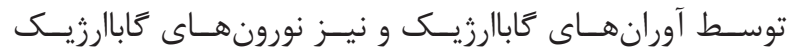

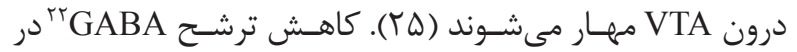

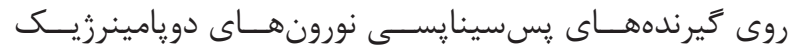

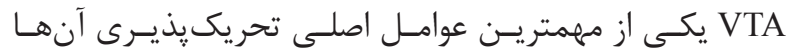

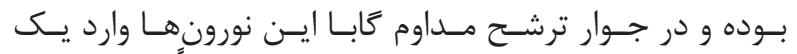

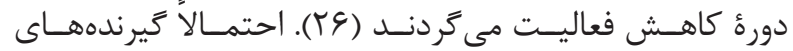

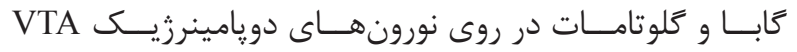

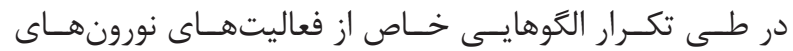

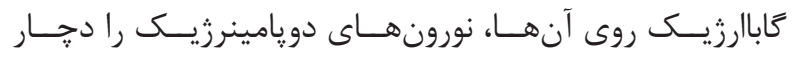

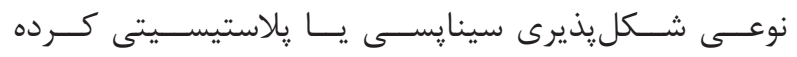

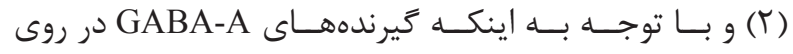

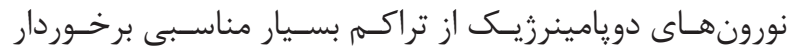

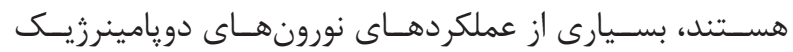

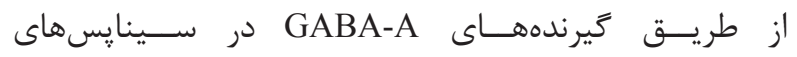

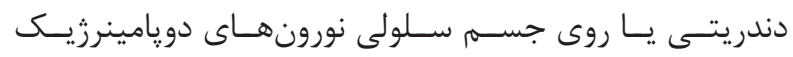

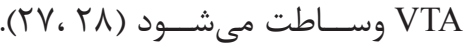

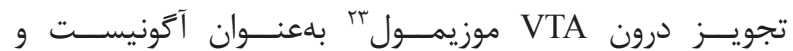

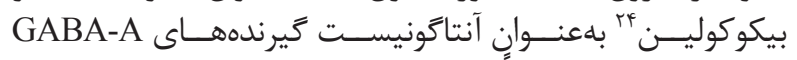

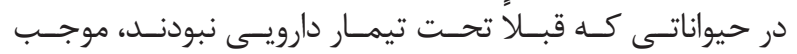

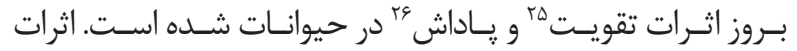

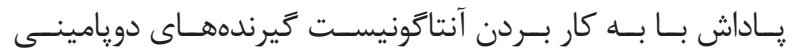

\footnotetext{
${ }^{1}$ Ventral tegmental area

${ }^{2}$ Limbic system

${ }^{3}$ Reward system

${ }^{4}$ Motivated behaviors

${ }^{5}$ Drug dependence

${ }^{6}$ Addiction

${ }^{7}$ Neuropsychiatric illnesses

${ }^{8}$ Dopaminergic

${ }^{9}$ GABAergic

${ }^{10}$ Glutamatergic

${ }^{11}$ Nucleus accumbens

${ }^{12}$ Prefrontal cortex

${ }^{13}$ Dopamine mesocorticolimbic
}

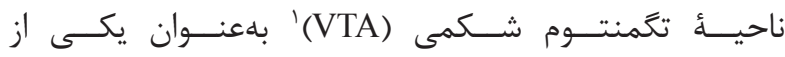

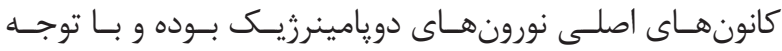

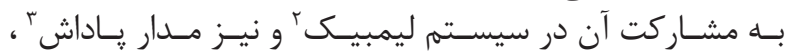

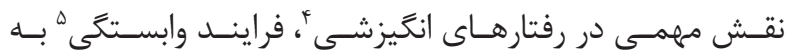

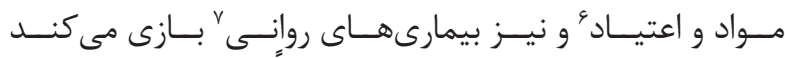

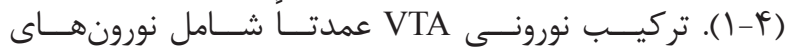

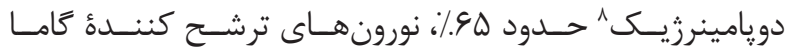

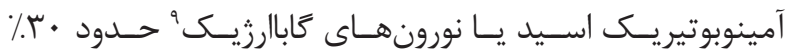

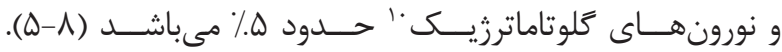

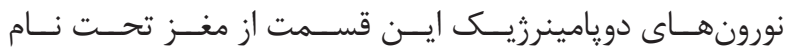

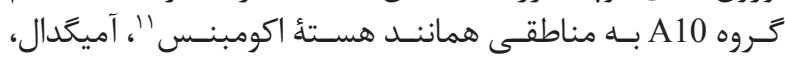

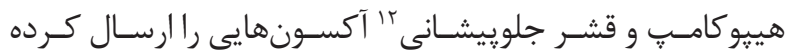

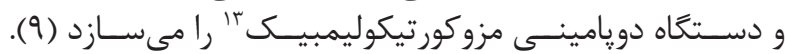

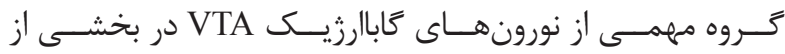

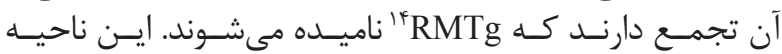

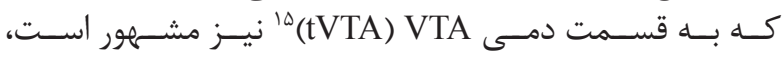

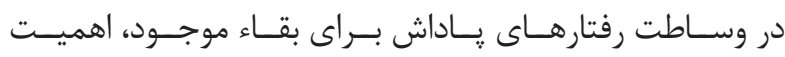
فراوانسى دارد (11 ، • (1).

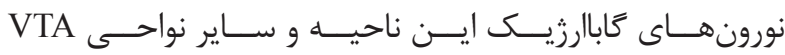

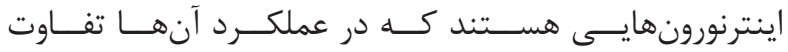

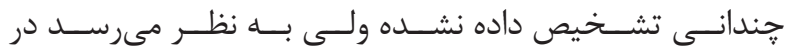

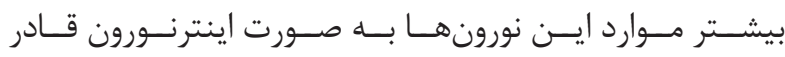

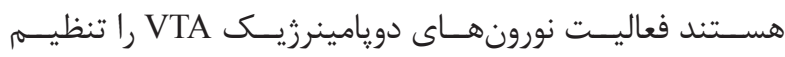

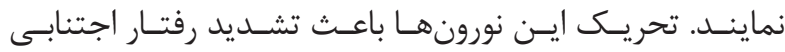

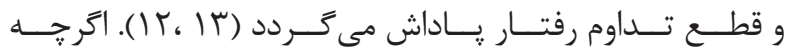

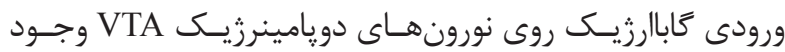

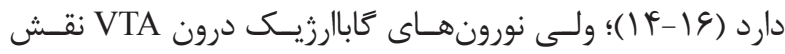

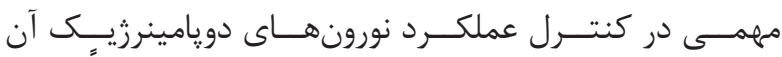

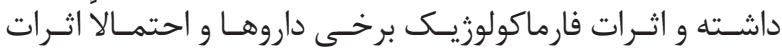

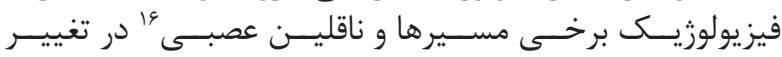

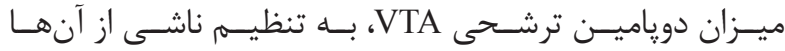

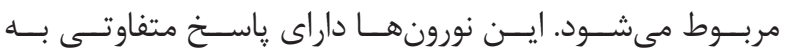

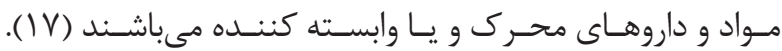

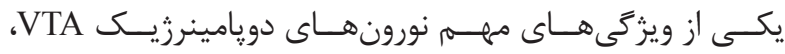

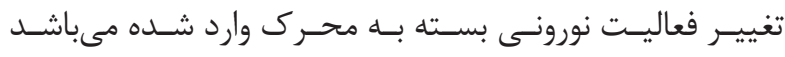

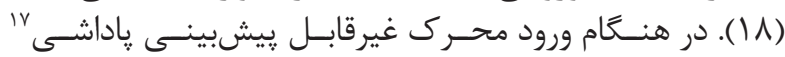

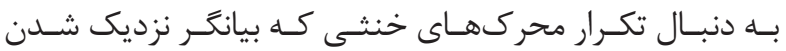

\footnotetext{
${ }^{14}$ Rostromedial tegmental nucleus

${ }^{15}$ Tail of the ventral tegmental area

${ }^{16}$ Neurotransmitters

${ }^{17}$ Unpredicted reward

${ }^{18}$ Regular

${ }^{19}$ Pacemaker

${ }^{20}$ Burst

${ }^{21}$ Simple-tonic

22 gamma-Aminobutyric acid

${ }^{23}$ Muscimol

${ }^{24}$ Bicuculline

${ }^{25}$ Reinforcing

${ }^{26}$ Rewarding
} 


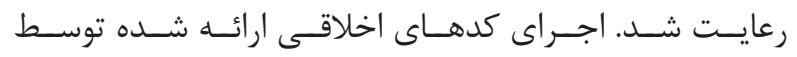

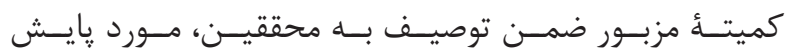

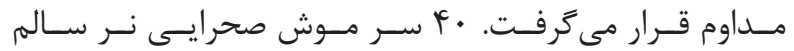

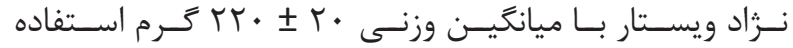

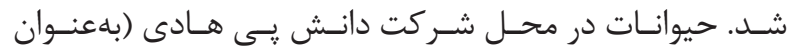

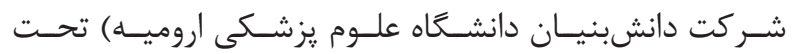

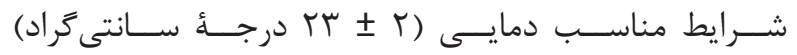

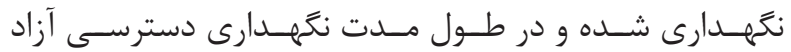

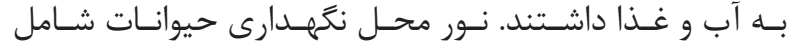

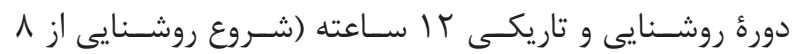

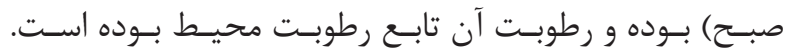

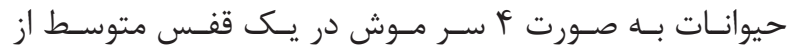

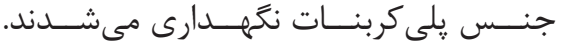

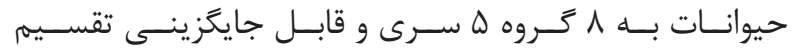

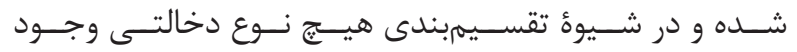

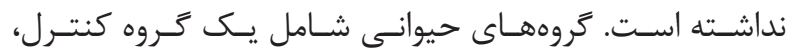

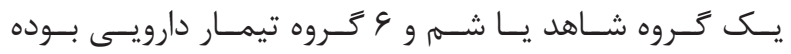

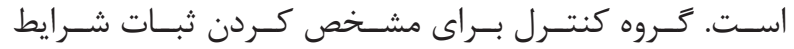

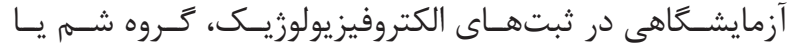

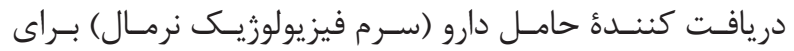

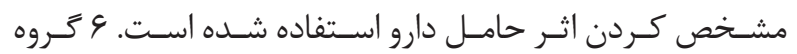

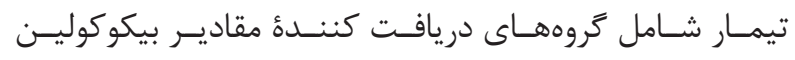

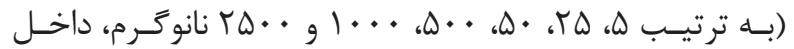

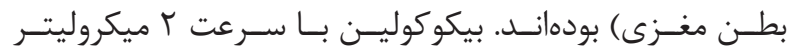

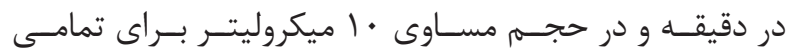

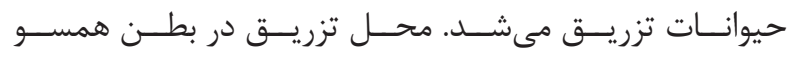

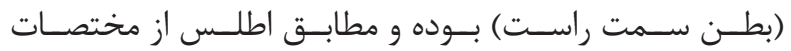

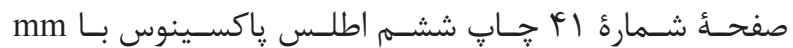

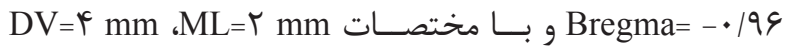

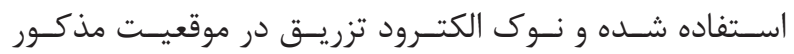

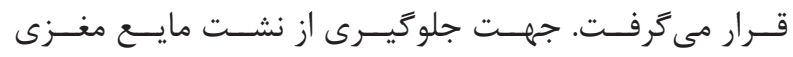

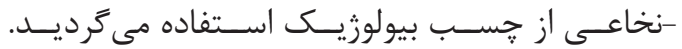

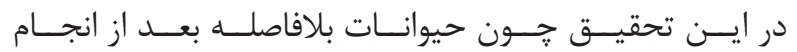

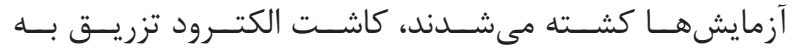

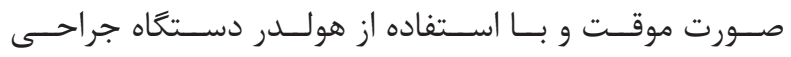

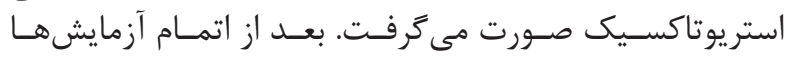

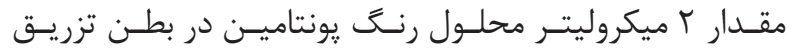

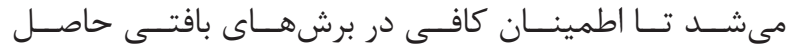

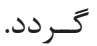

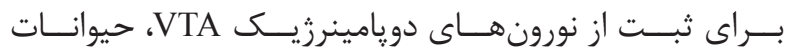

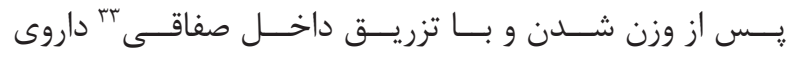

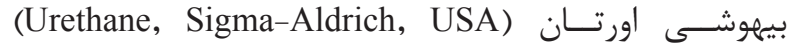

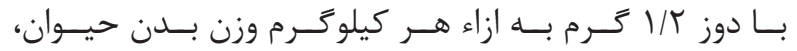

\section{${ }^{27}$ Disinhibition}

${ }^{28}$ Spike timing-dependent plasticity

${ }^{29} \mathrm{~N}$-methyl-D-aspartate receptor

${ }^{30}$ Burst firings

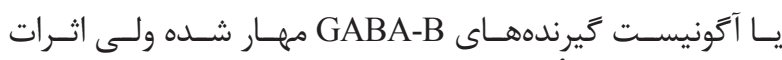

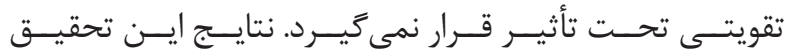

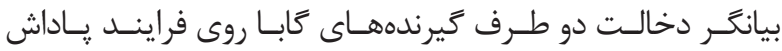

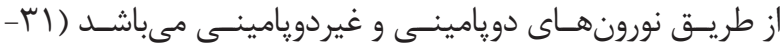

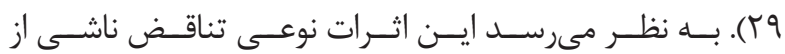

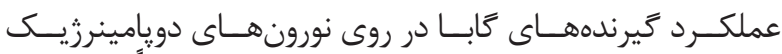

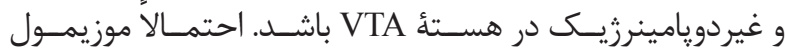

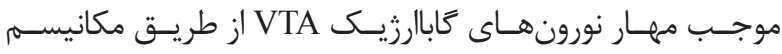

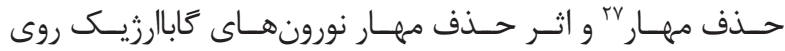

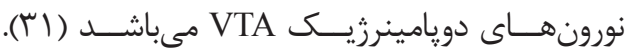

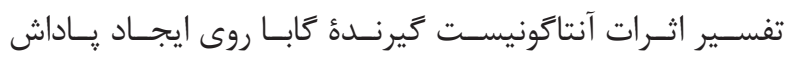

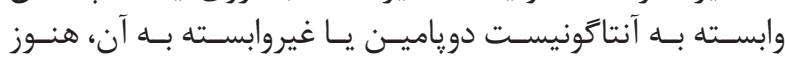

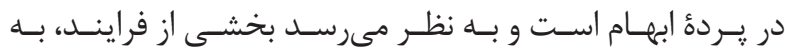

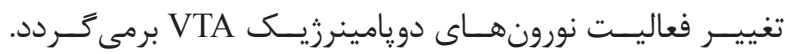

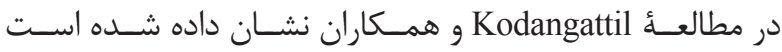

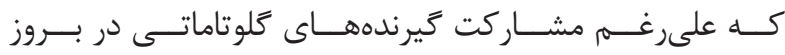

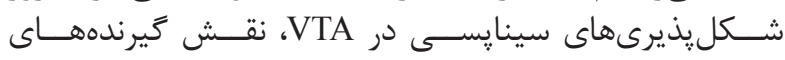

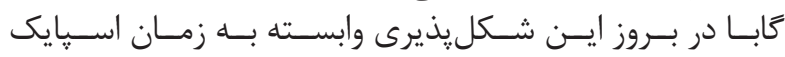

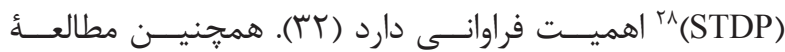

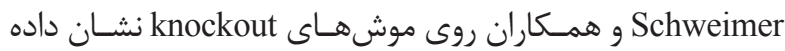

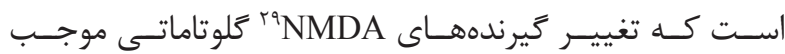

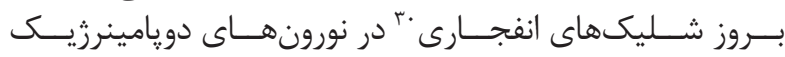

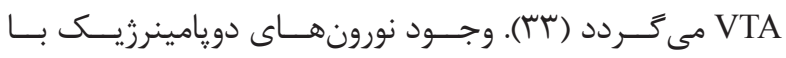

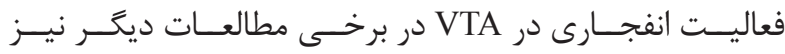

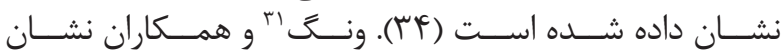

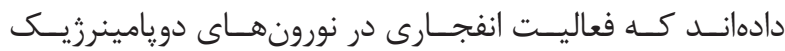

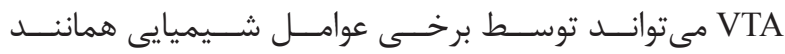

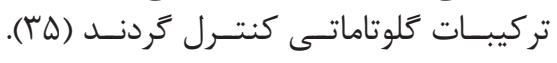

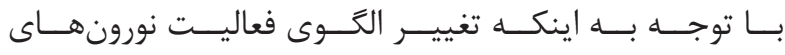

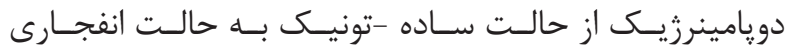

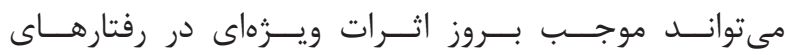

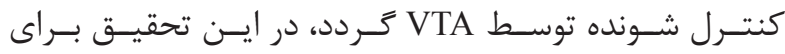

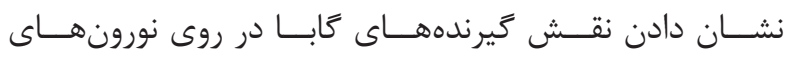

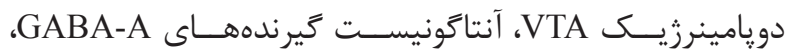

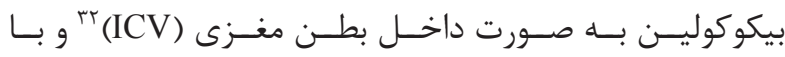

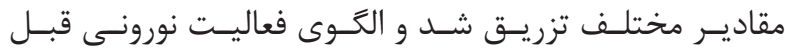

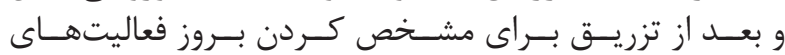

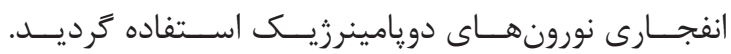

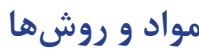

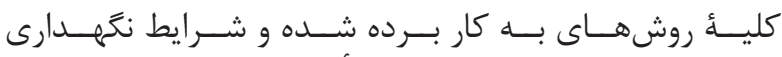

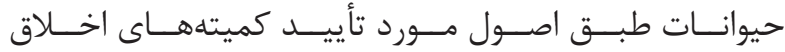

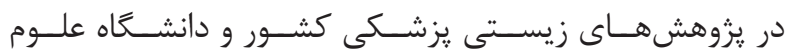

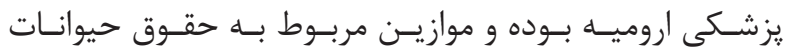

${ }^{31}$ Wang
${ }^{32}$ Intracerebroventricular
${ }^{33}$ Intraperitoneal injection 


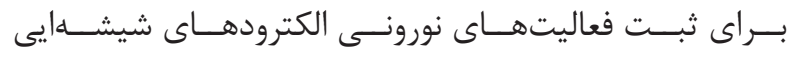

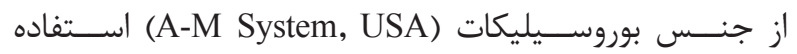

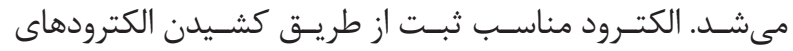

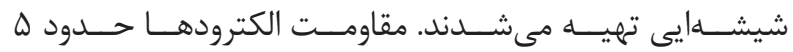

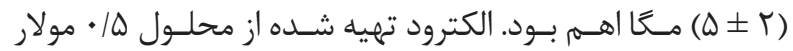

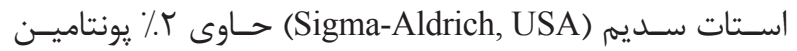

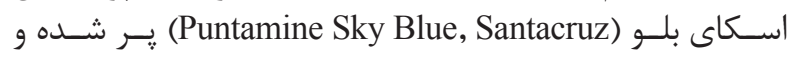

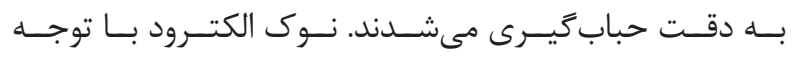

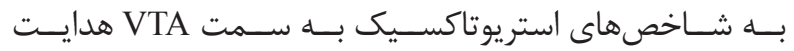

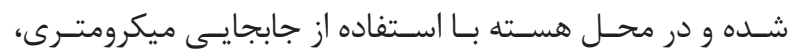

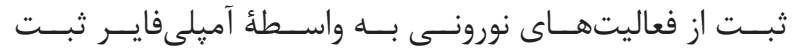

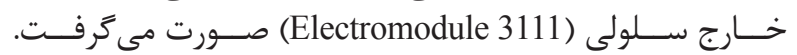

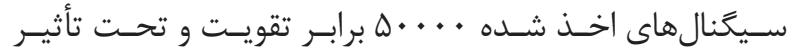

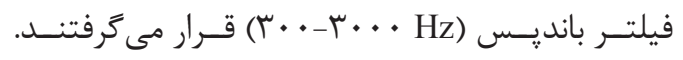

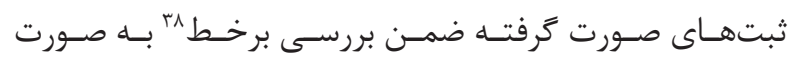

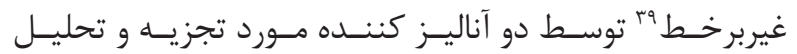

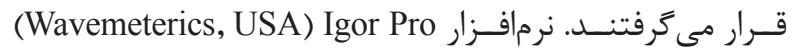

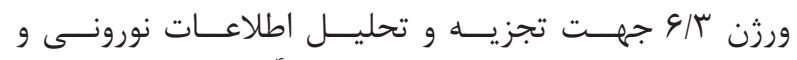

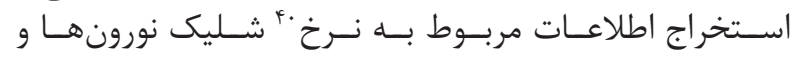

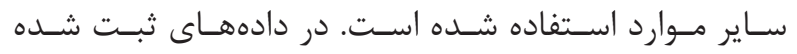

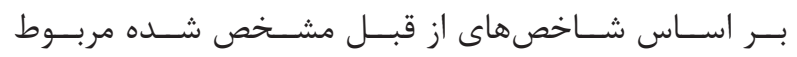

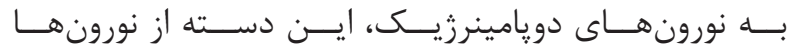

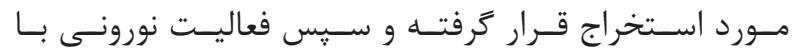

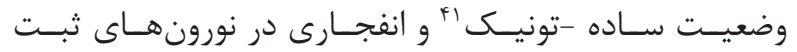

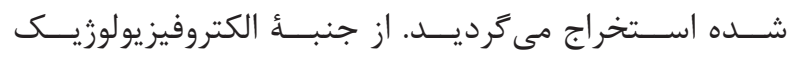

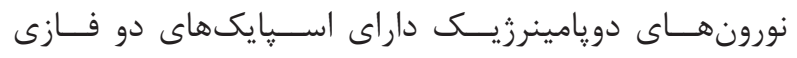

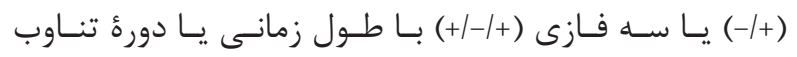

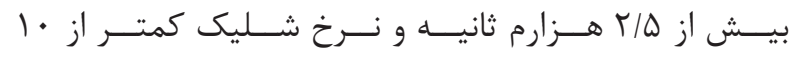

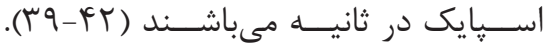

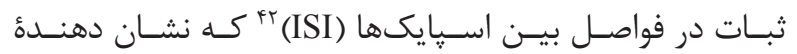

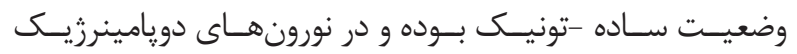

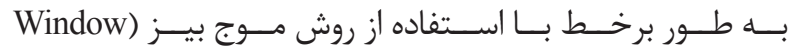

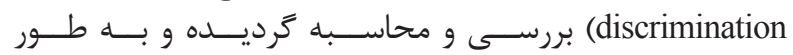

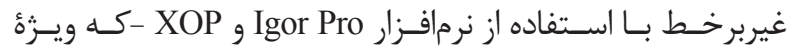

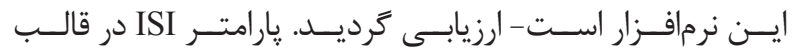

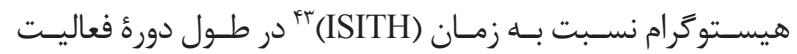

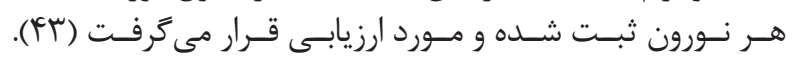

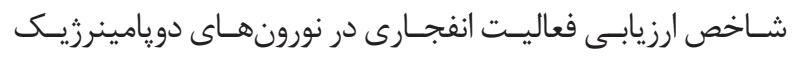

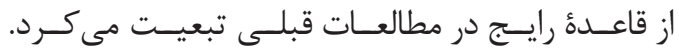

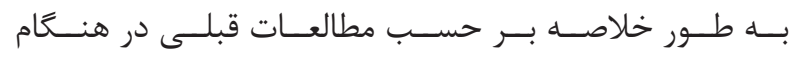

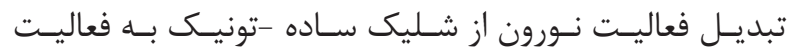

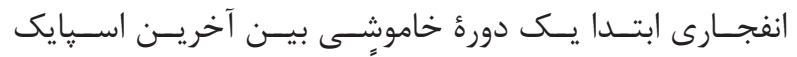

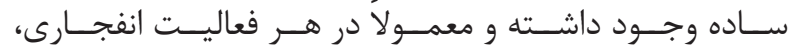

\footnotetext{
${ }^{34}$ Booster

${ }^{35}$ Interaural axis

${ }^{36}$ Bregma zero-zero

${ }^{37}$ Single neuron recording

${ }^{38}$ On-line
}

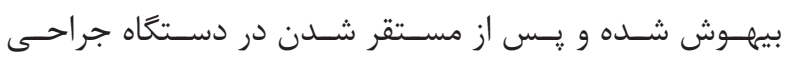

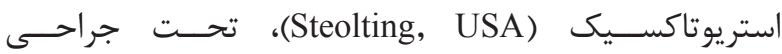

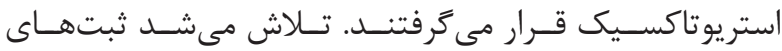

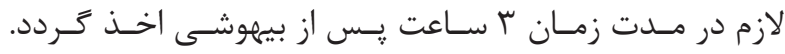

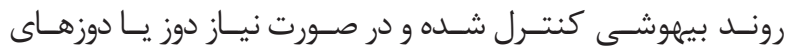

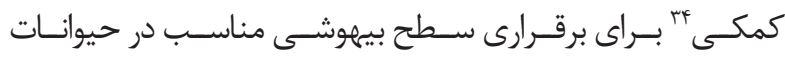

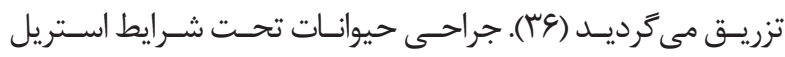

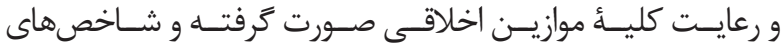

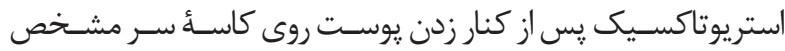

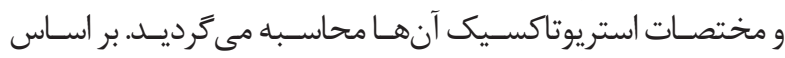

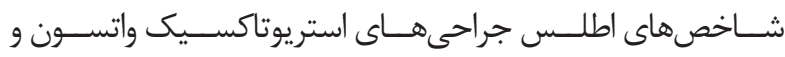

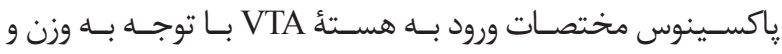

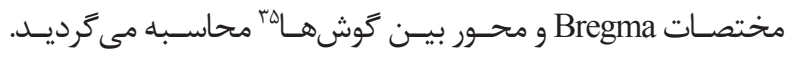

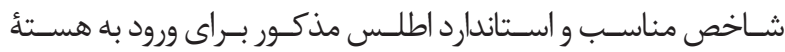

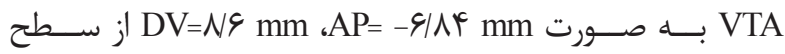

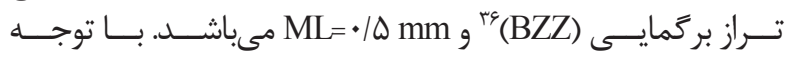

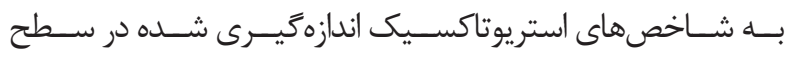

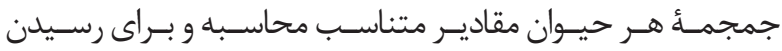

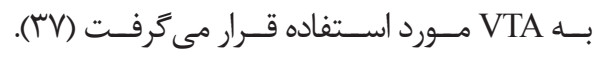

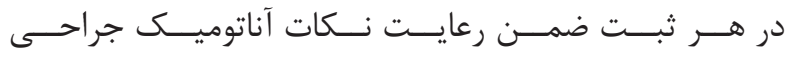

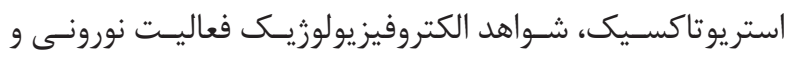

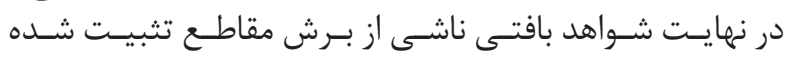

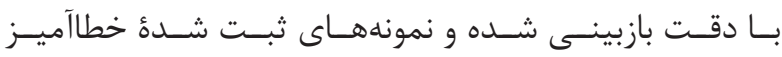

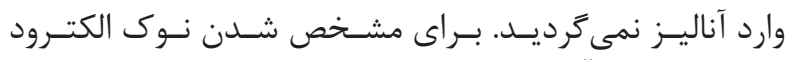

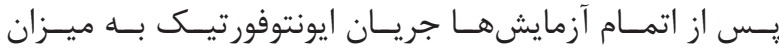

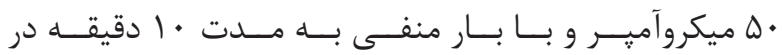

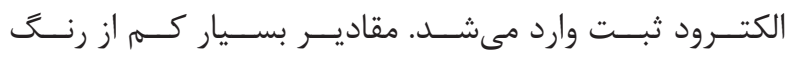

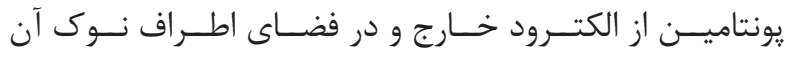

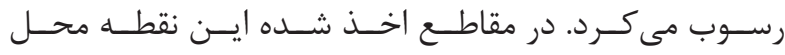

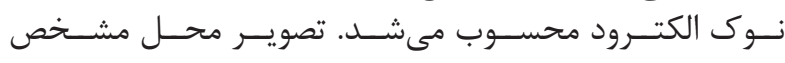

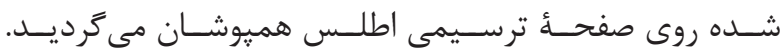

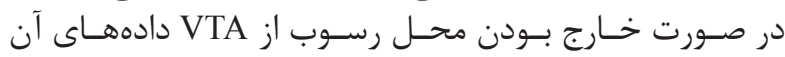

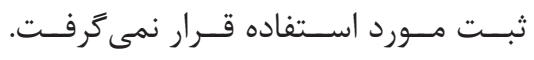

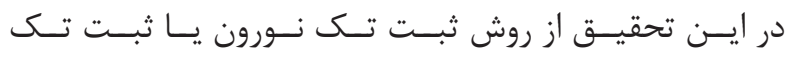

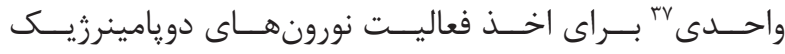

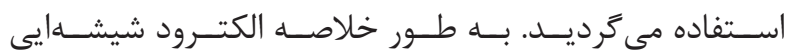

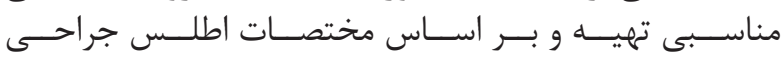

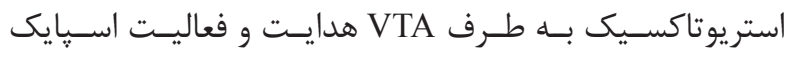

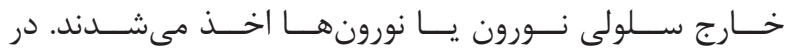

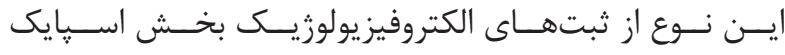

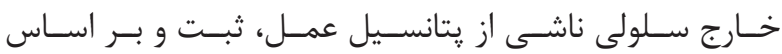

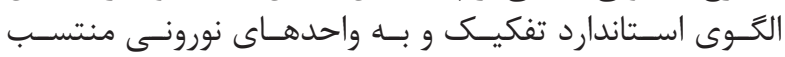

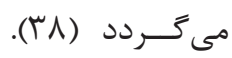

\footnotetext{
${ }^{39}$ Off-line

${ }^{40}$ Rate

${ }^{41}$ Simple-tonic mode

${ }^{42}$ Inter spike interval

${ }^{43}$ Inter spike interval time histogram
} 


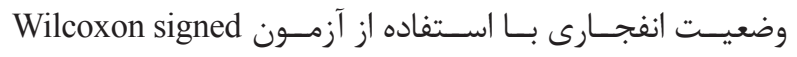

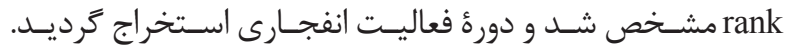

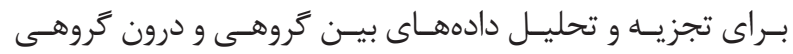

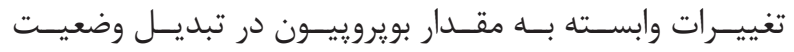

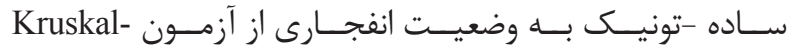
دادها Wallis

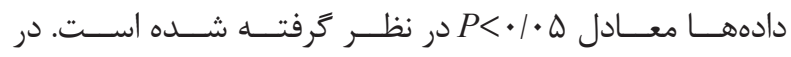

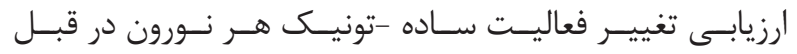

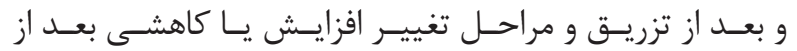

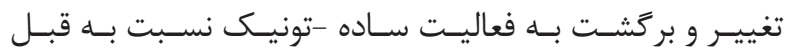

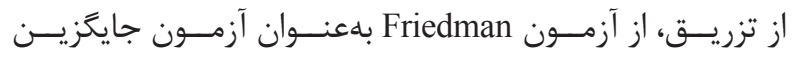

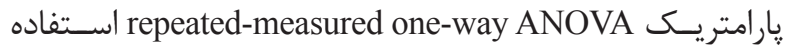

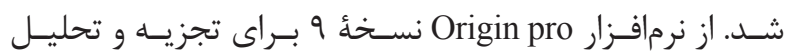

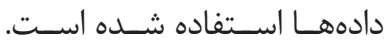

بافته ها

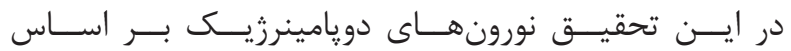

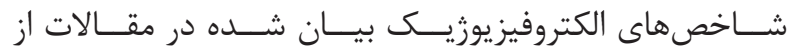

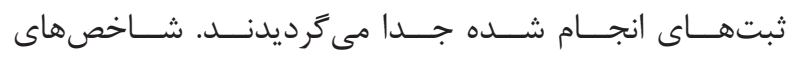

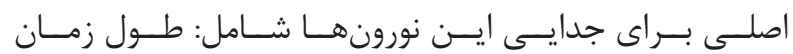

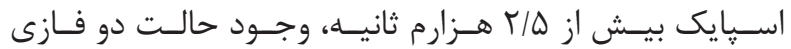

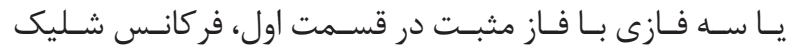

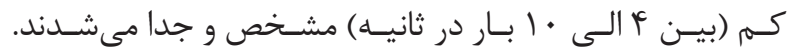

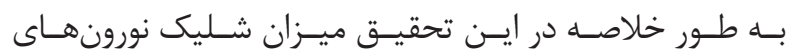

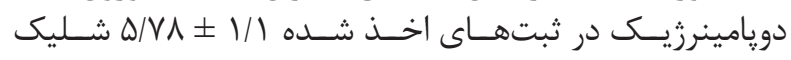

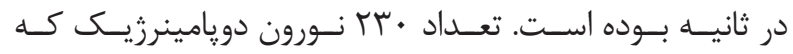

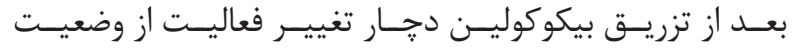

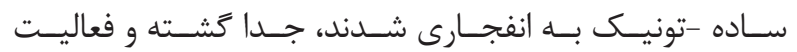

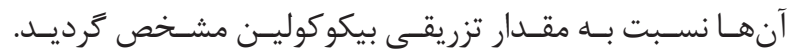

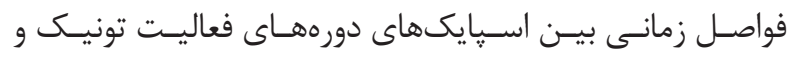

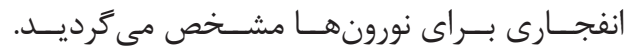

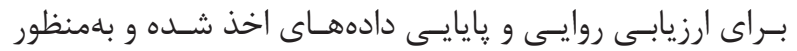

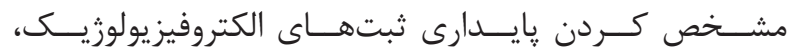

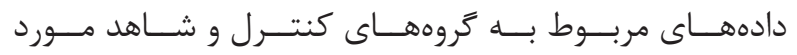

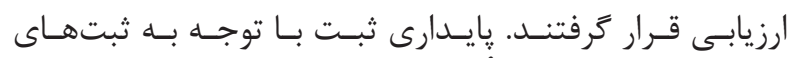

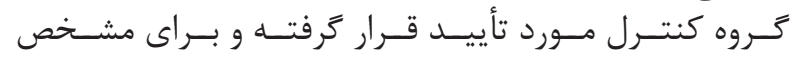

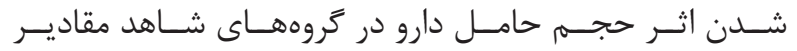

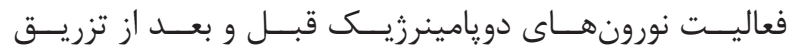

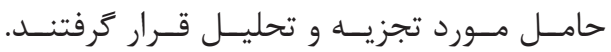

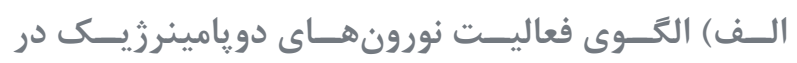
كَـروه شـاهد

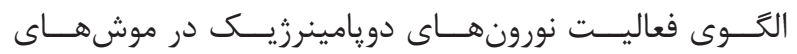

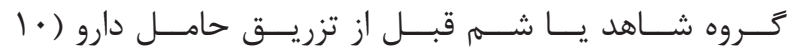

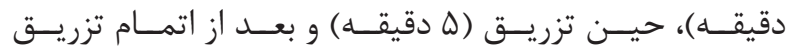

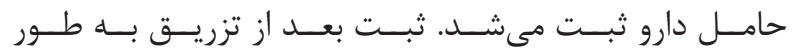

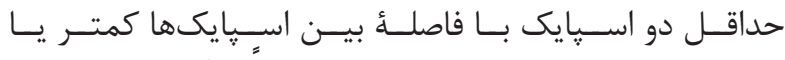

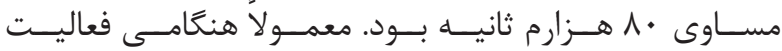

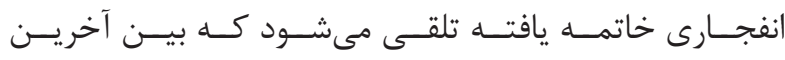

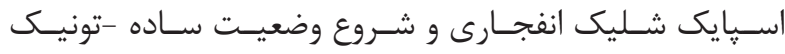

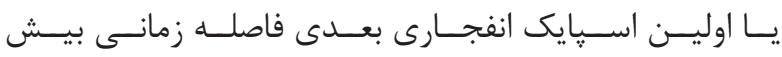

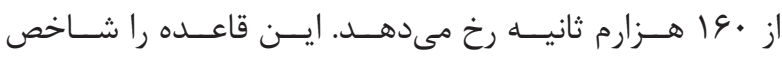

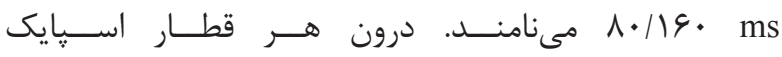

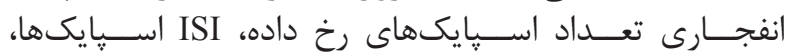

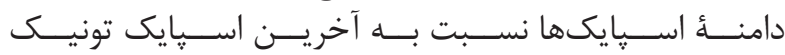

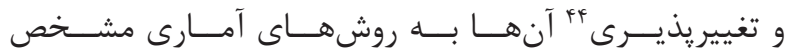

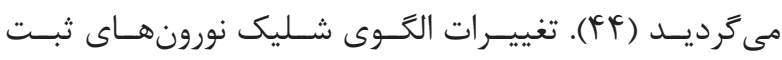

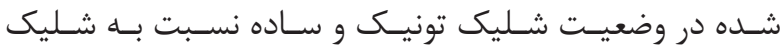

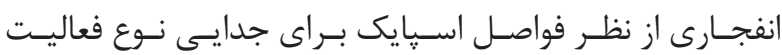

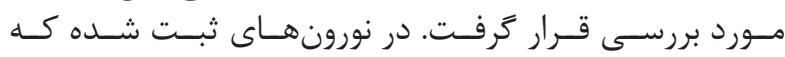

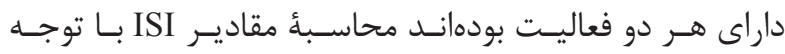

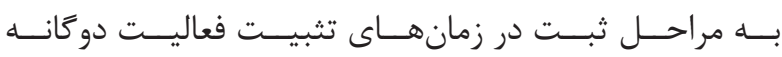

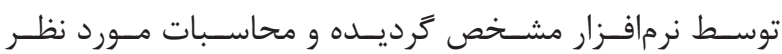

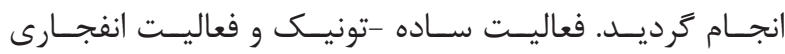

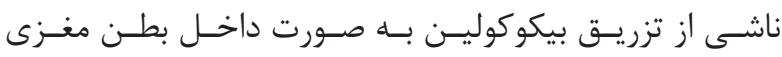

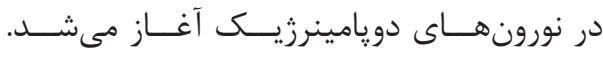

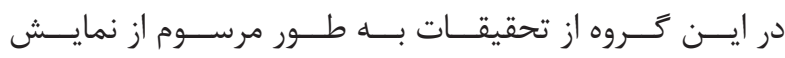

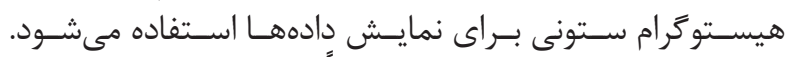

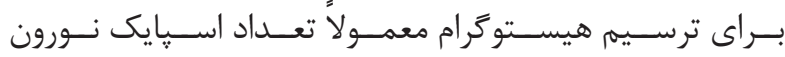

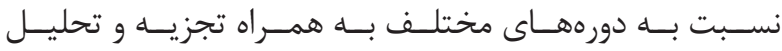

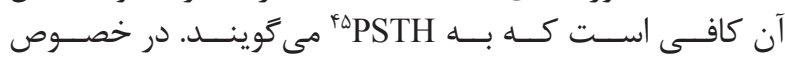

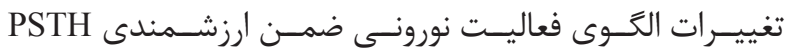

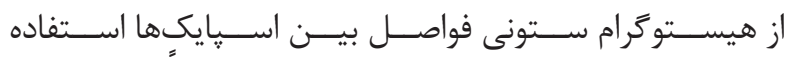

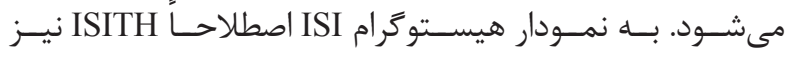

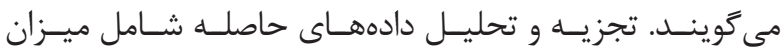

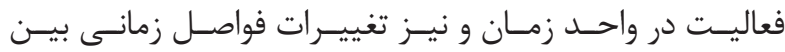

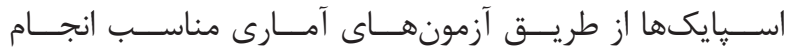

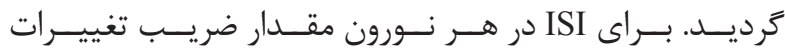
(CV)

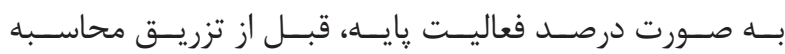

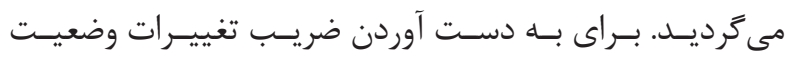

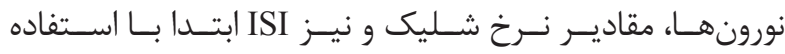

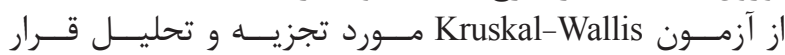

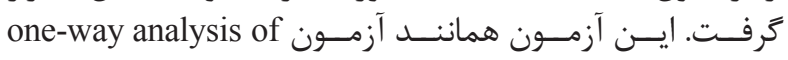

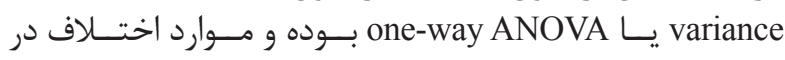

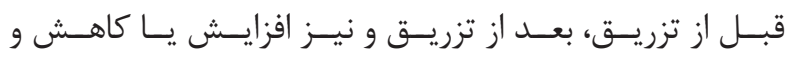

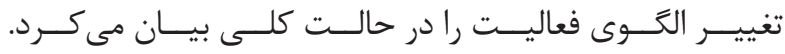

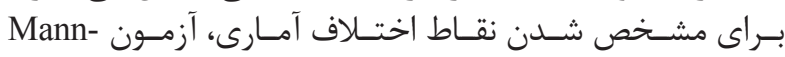
PSTH بــــ Whitney U

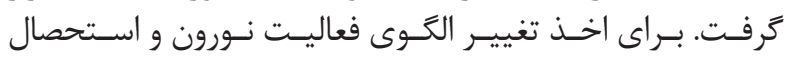

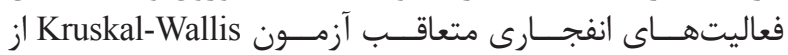

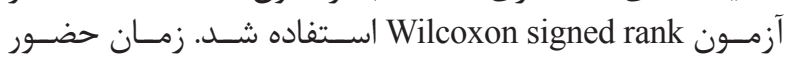

${ }^{44}$ Variability

${ }^{45}$ Peri-stimulus time histogram

${ }^{46}$ Coefficient of variation 
نــورون دويامينرزيـك آورده شـده اسـت.

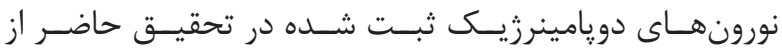

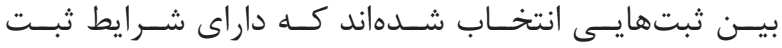

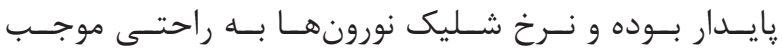

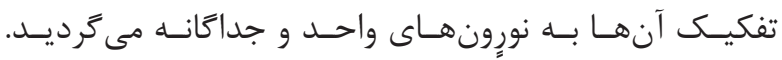

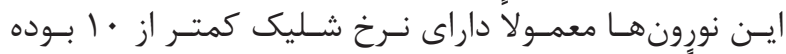

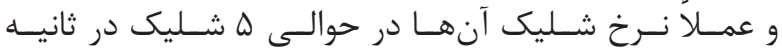

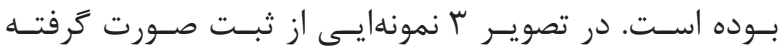

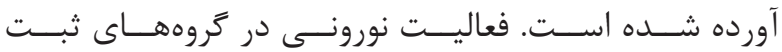

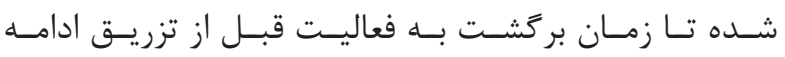

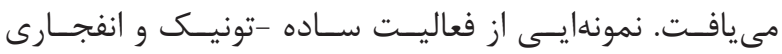

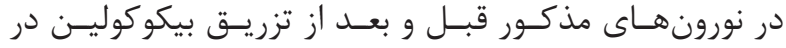

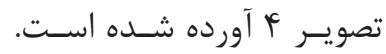

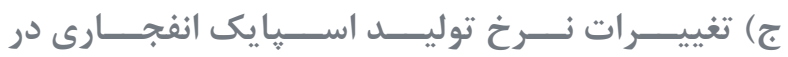

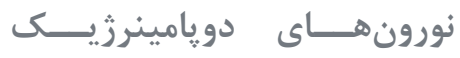

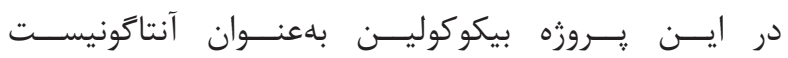

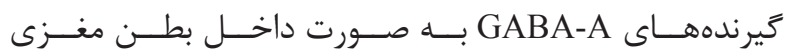

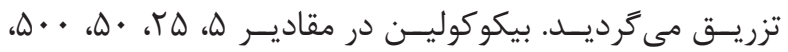

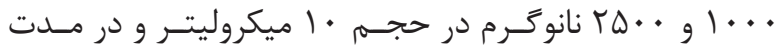

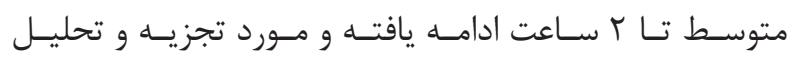

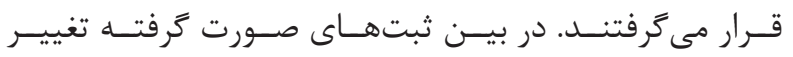

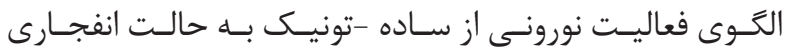

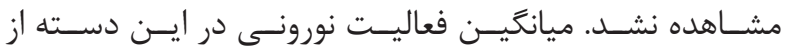

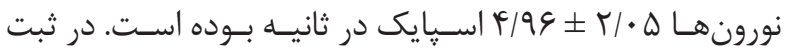

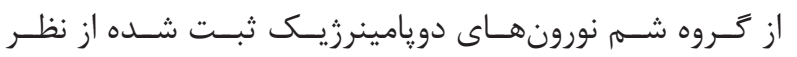

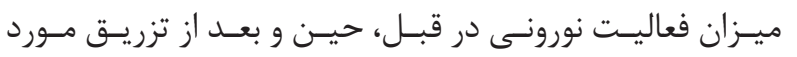

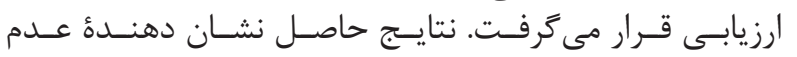

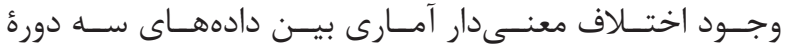

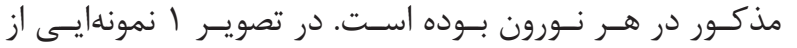

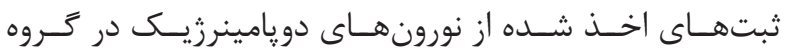

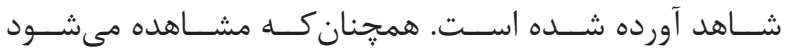

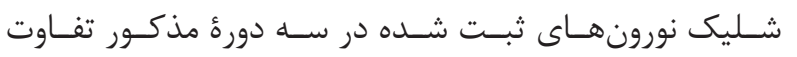

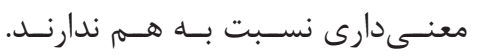

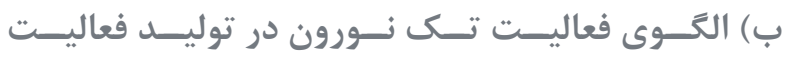

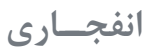

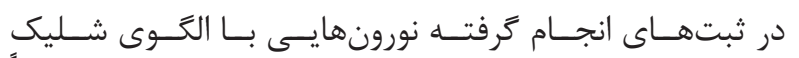

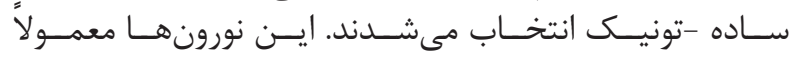

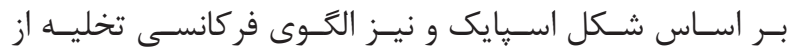

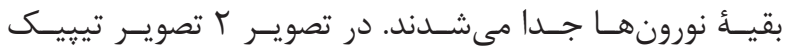

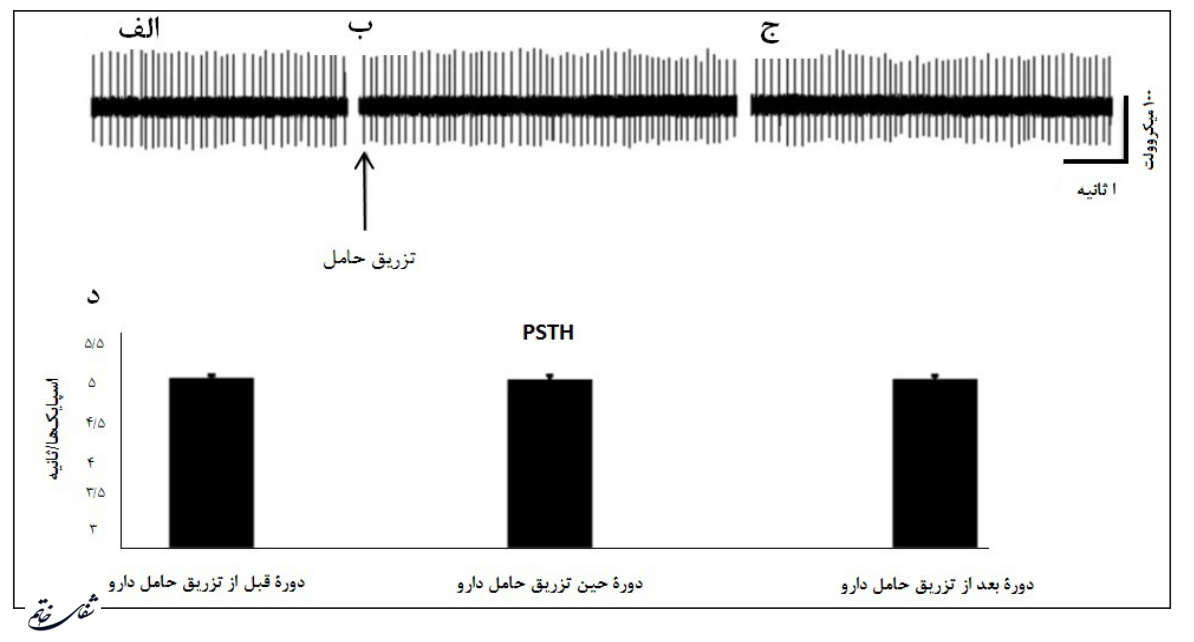

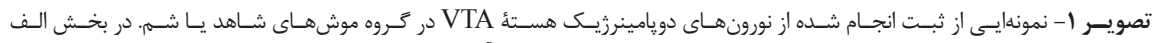

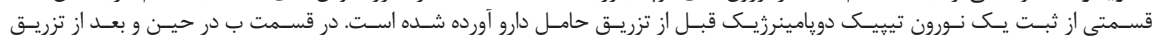

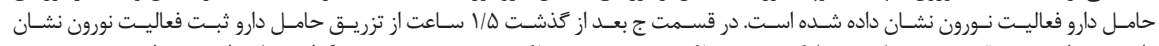

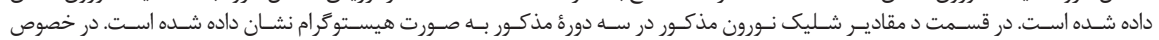

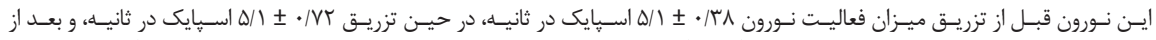

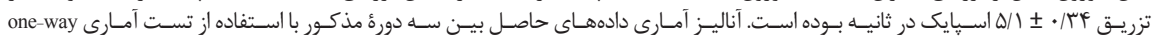
repeated measured ANOVA

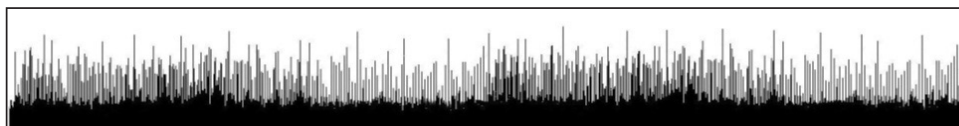

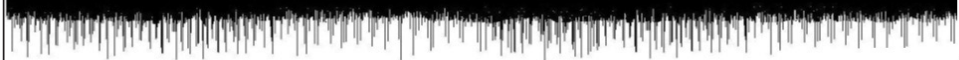

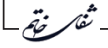

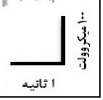

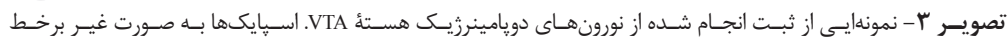

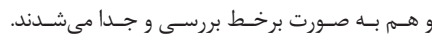

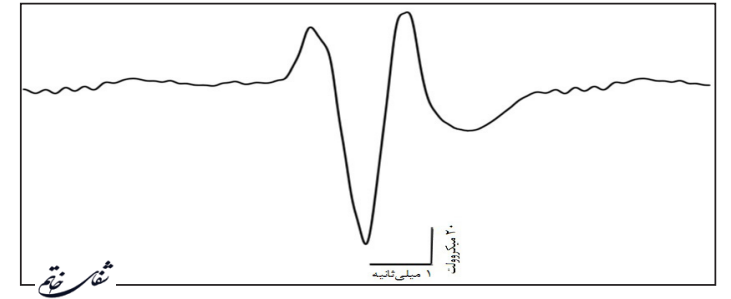

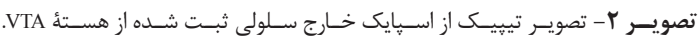

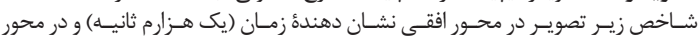

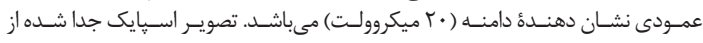

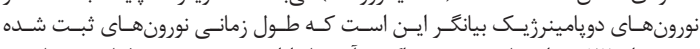

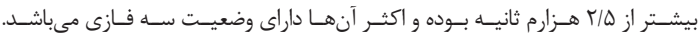




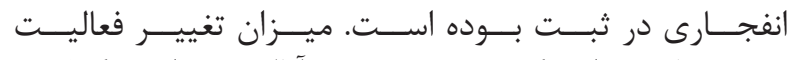

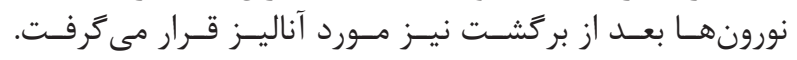

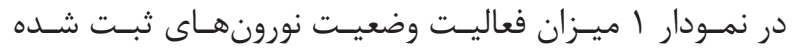

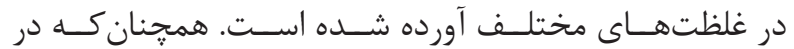

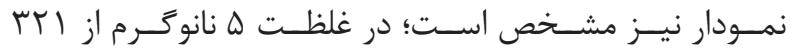

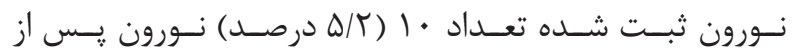

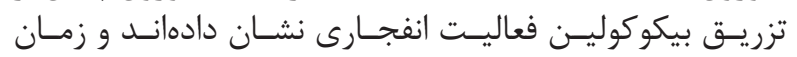

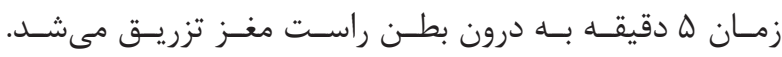

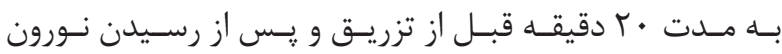

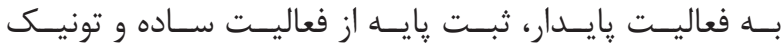

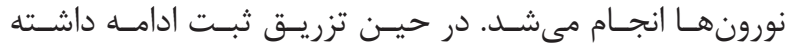

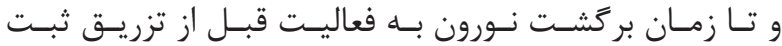

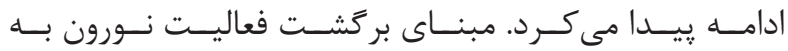

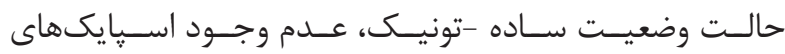

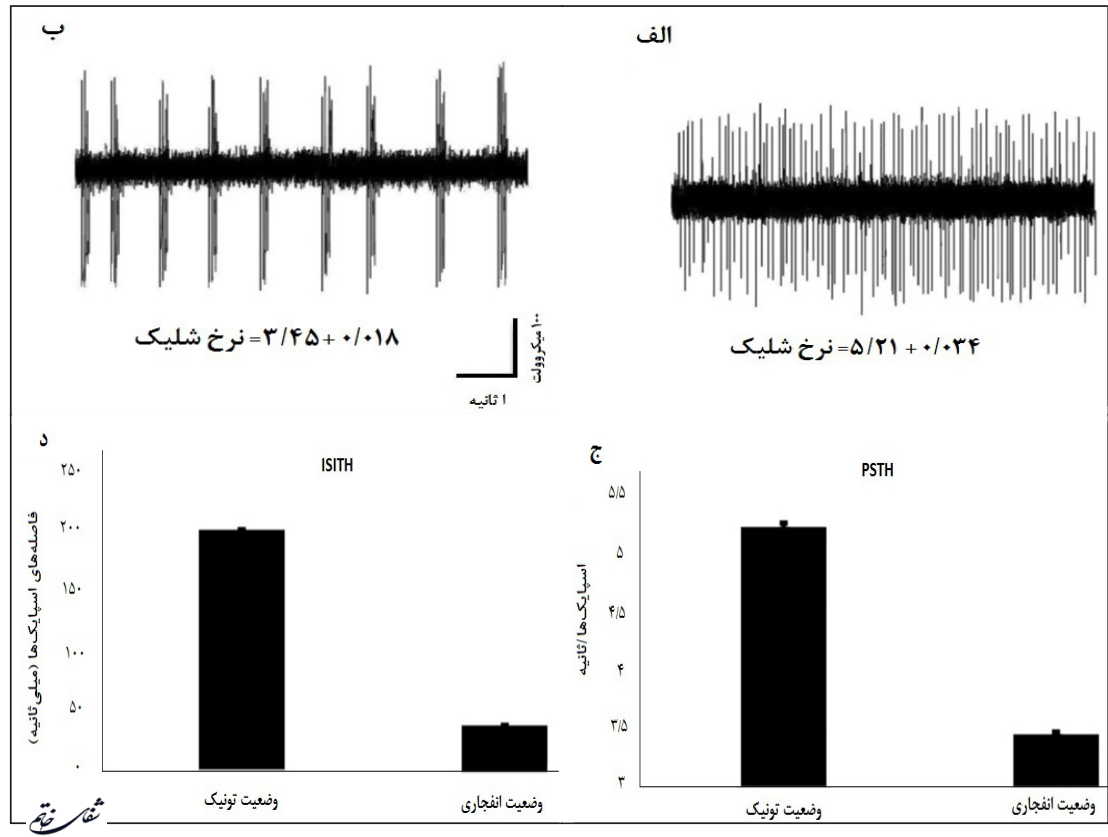

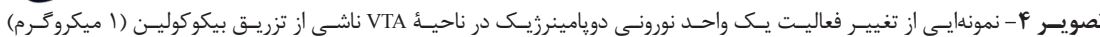

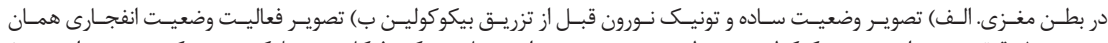

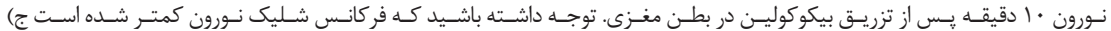

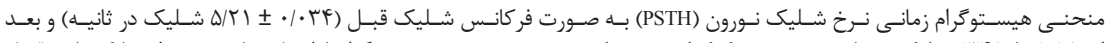

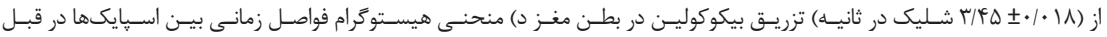

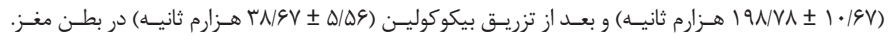
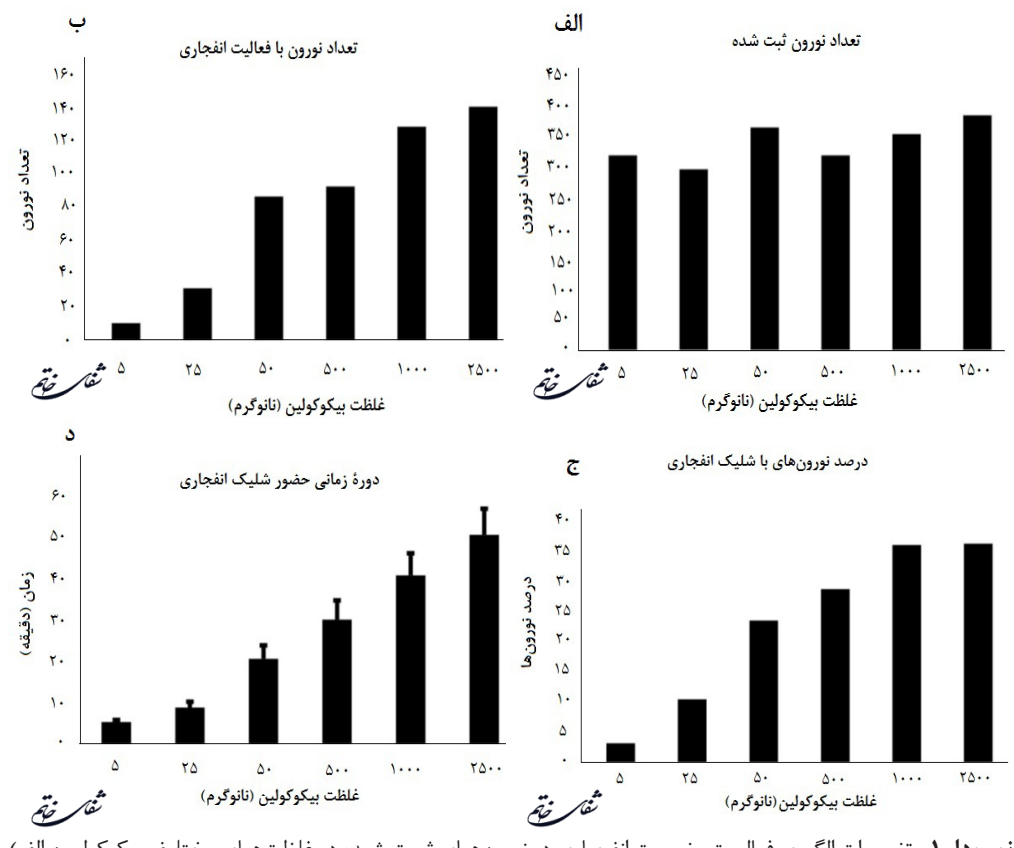

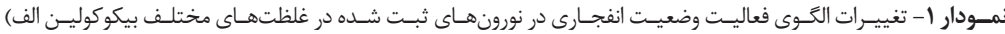

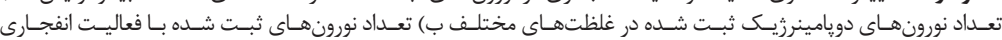

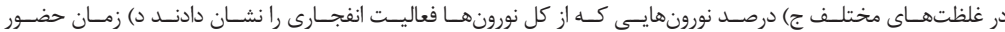

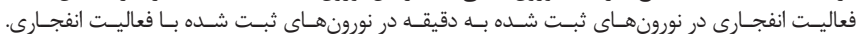


بحث و نتيجه كيرى

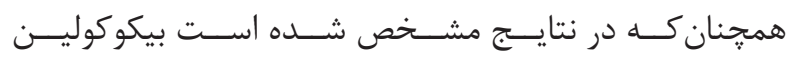

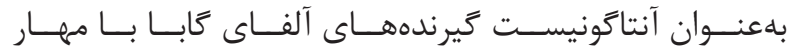

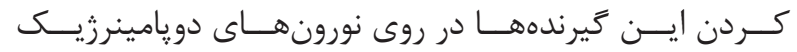

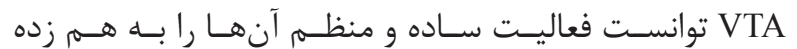

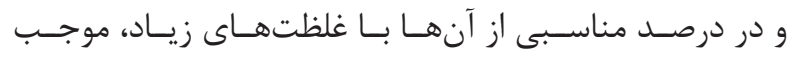

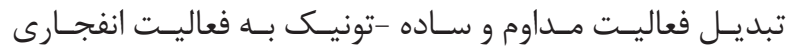

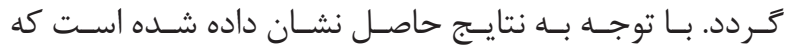

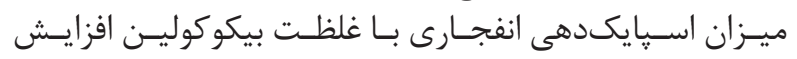

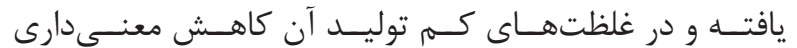

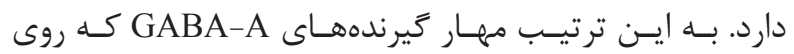

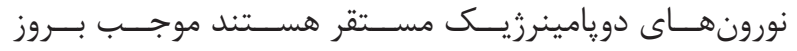

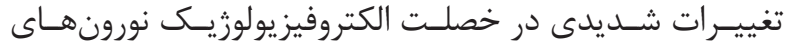

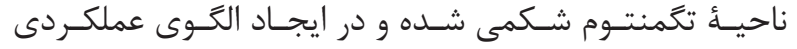

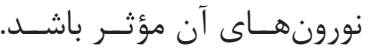

نورونهـاى دويامينرزيــ VTA داراى شـليك نورونسى تونيـك

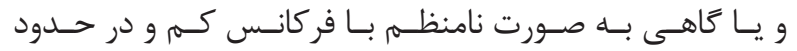

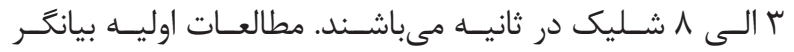

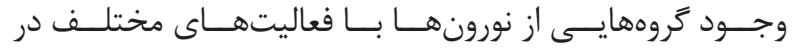

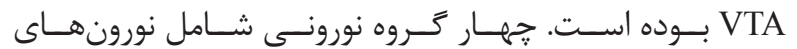

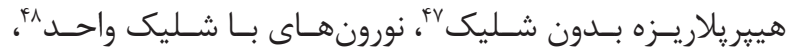

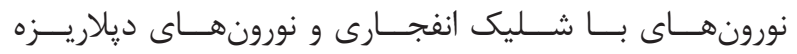

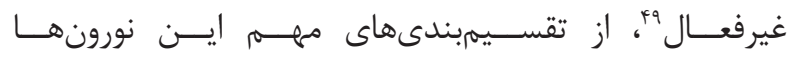

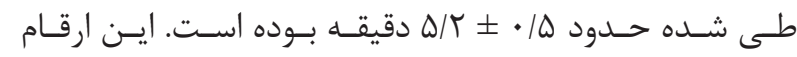

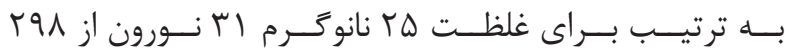

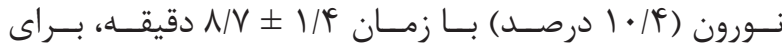

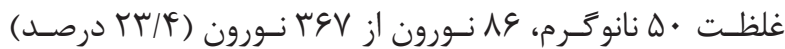

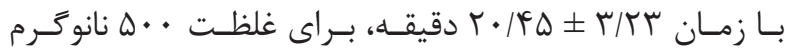

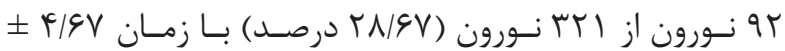

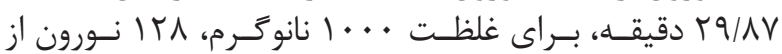

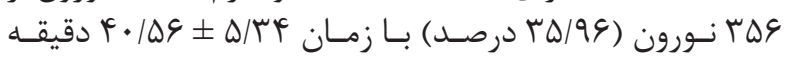

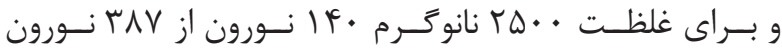

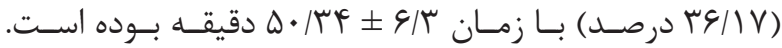

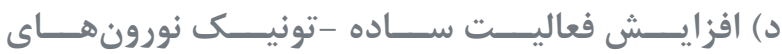

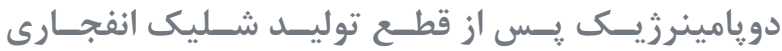

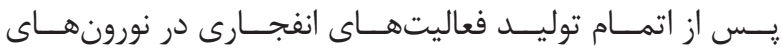

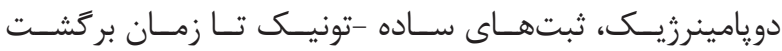

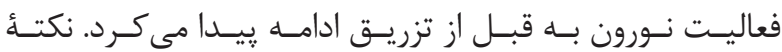

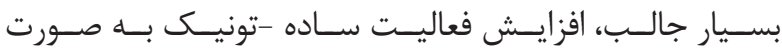

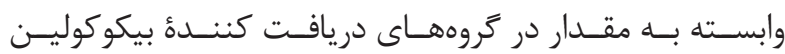

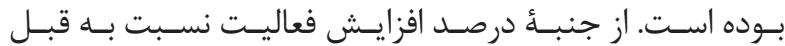

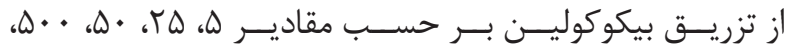

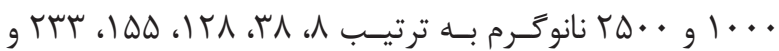

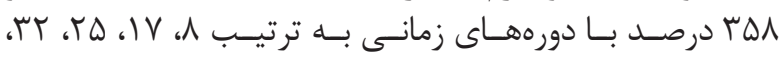

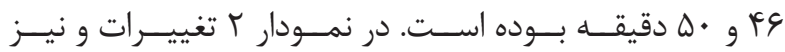

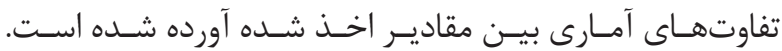

الف ميزان فعاليت قبل از تزريق
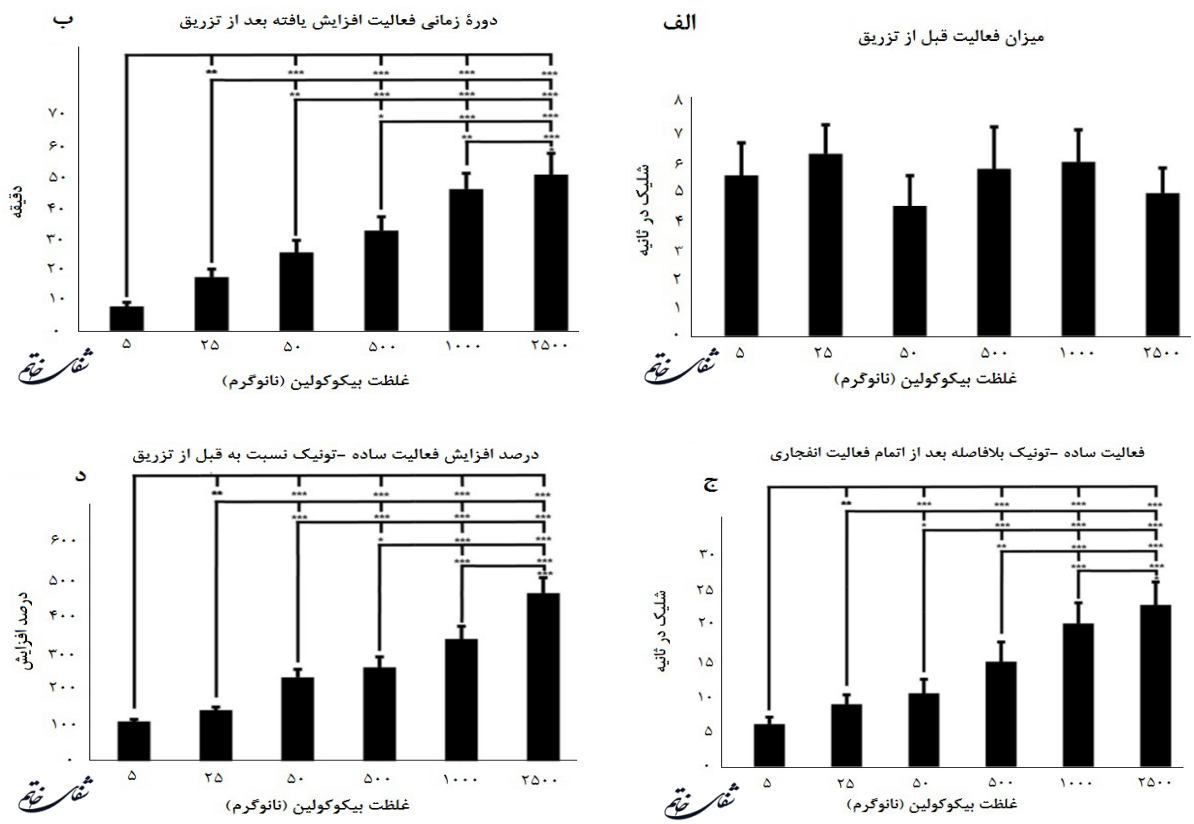

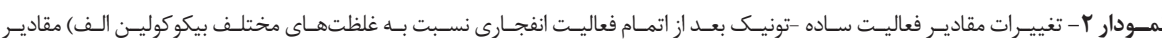

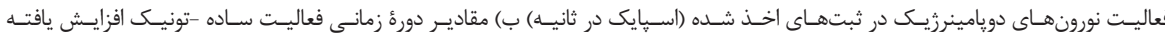

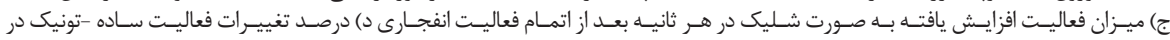

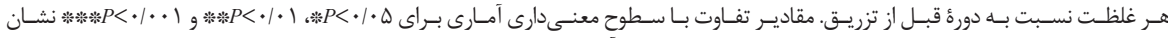

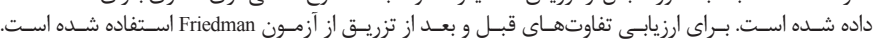

${ }^{47}$ Hyperpolarized, non-firing

${ }^{48}$ Single spike firing

${ }^{49}$ Depolarization inactivation 


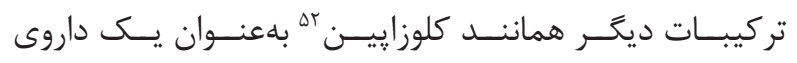

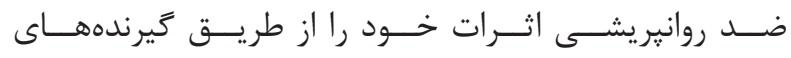

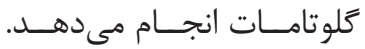

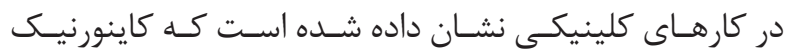

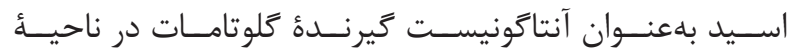

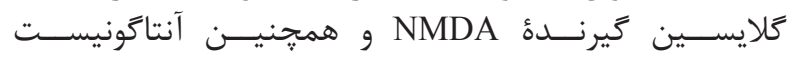

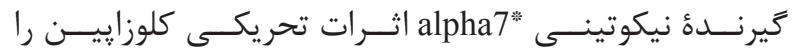

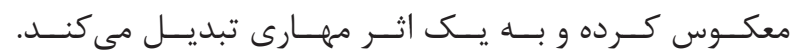

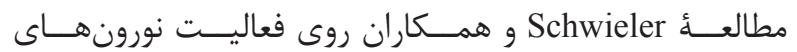

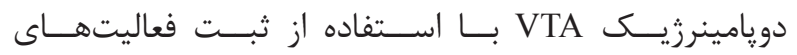

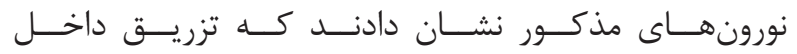

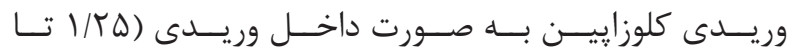

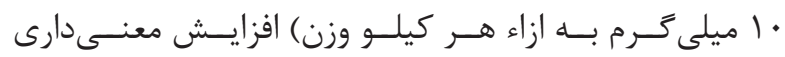

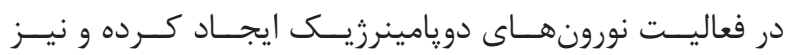

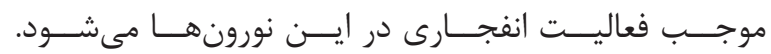

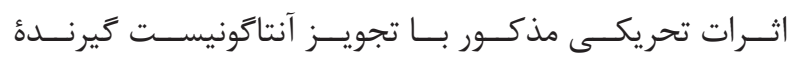

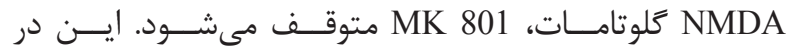

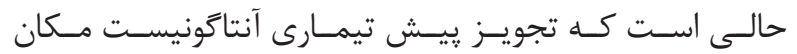

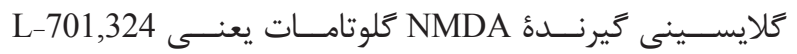

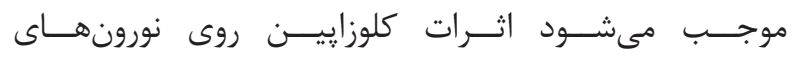

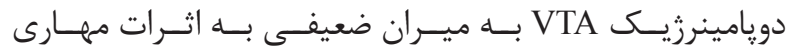

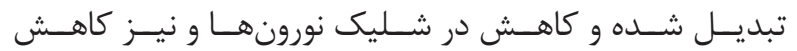

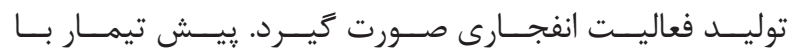

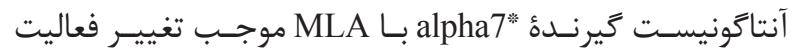

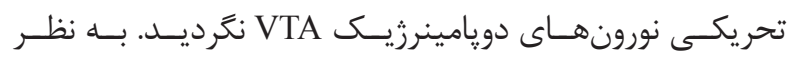

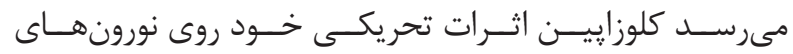

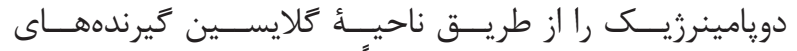

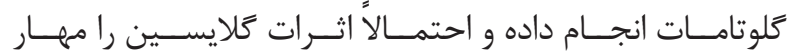

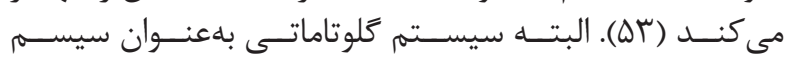

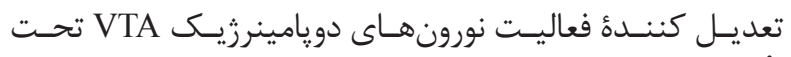

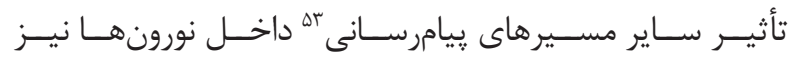

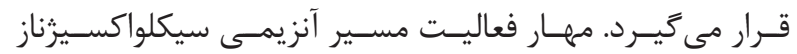

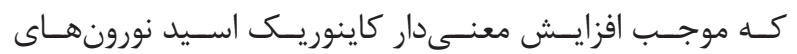

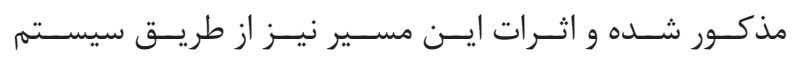
كلوتاماتـى تعديــل مى شـــود (Af)

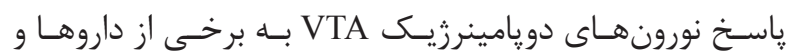

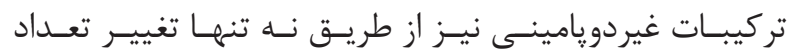

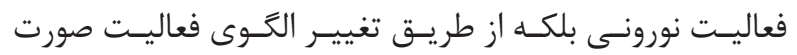

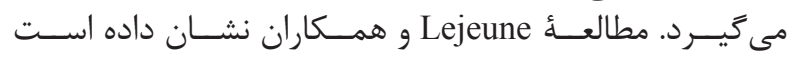

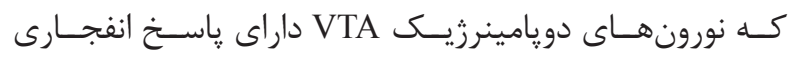

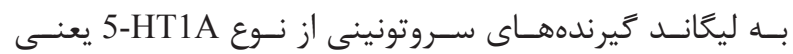

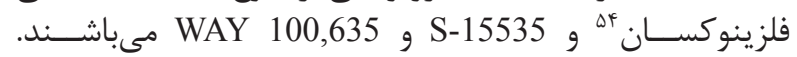

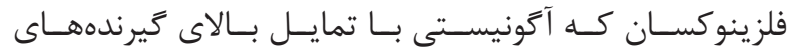

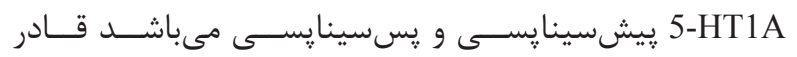

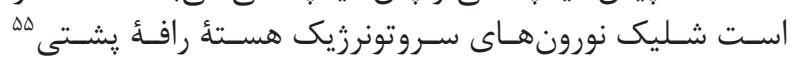

${ }^{50}$ Chloral hydrate

${ }^{51}$ Kynurenic

${ }^{52}$ Clozapine

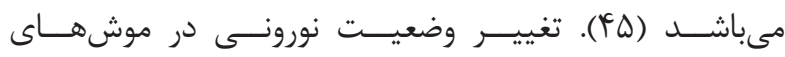

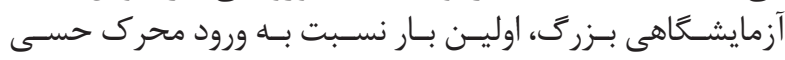

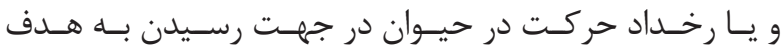

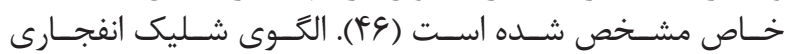

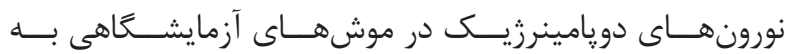

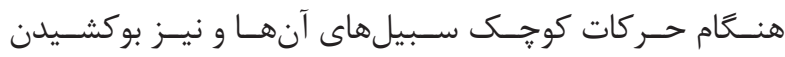

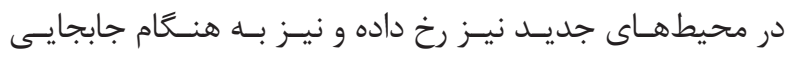

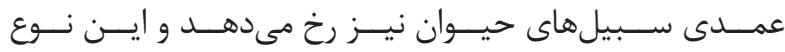

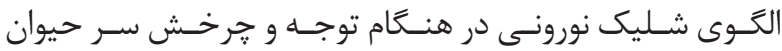

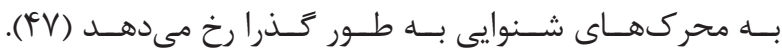

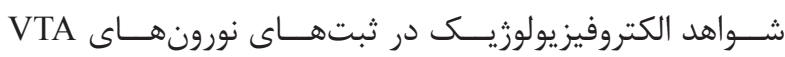

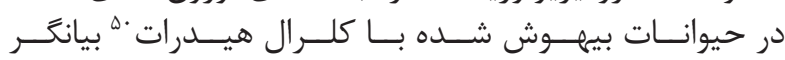

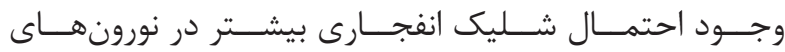

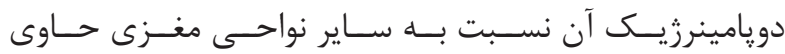

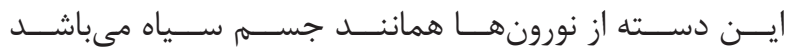

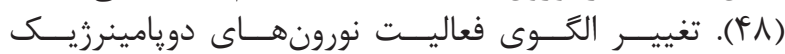

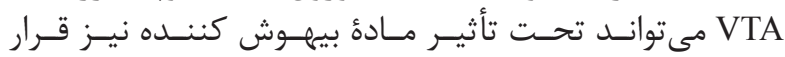

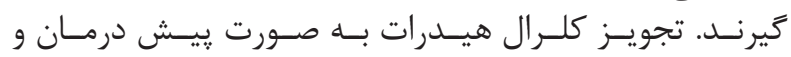

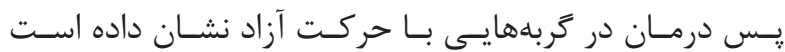

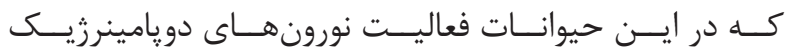

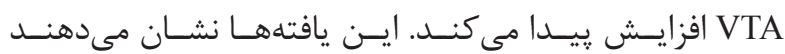

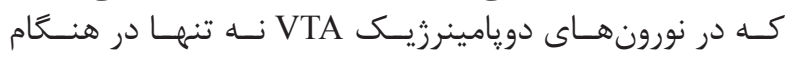

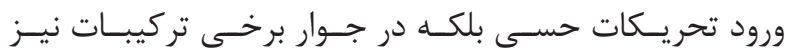

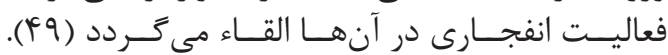

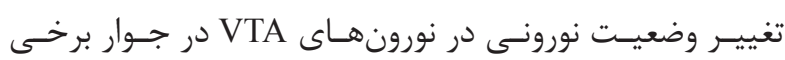

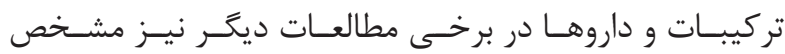

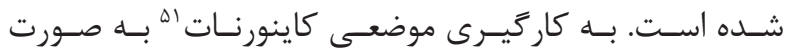

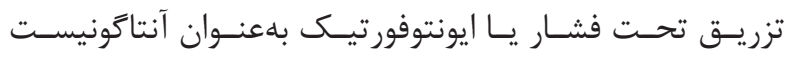

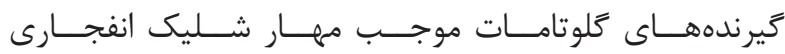

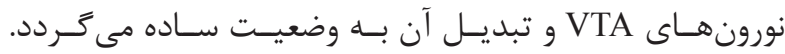

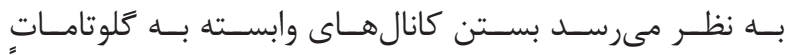

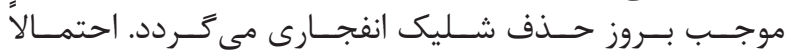

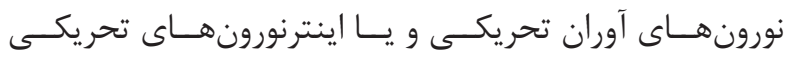

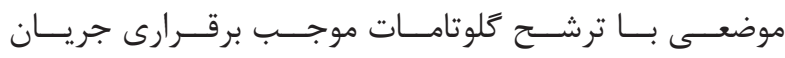

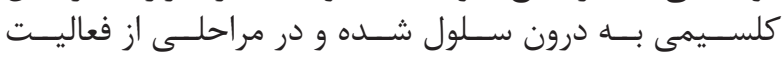

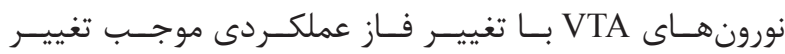

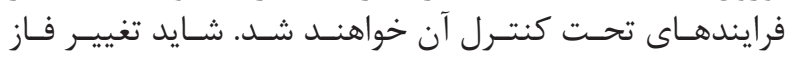

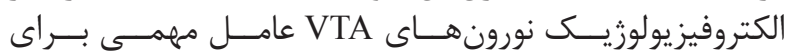

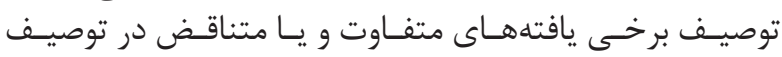

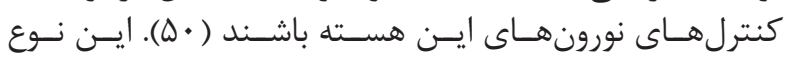

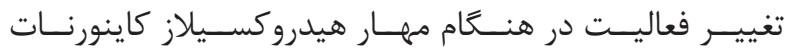

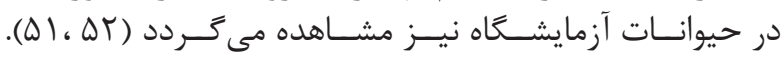

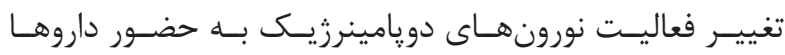

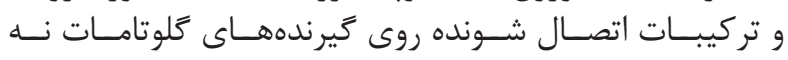

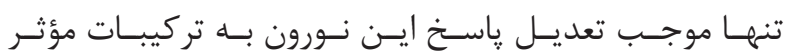

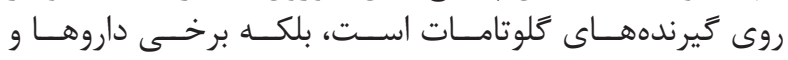

\footnotetext{
${ }^{53}$ Signaling

${ }^{54}$ Flesinoxan

${ }^{55}$ Dorsal raphe nucleus
} 


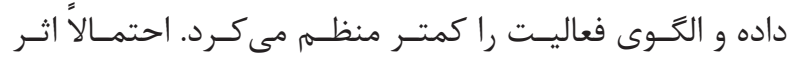

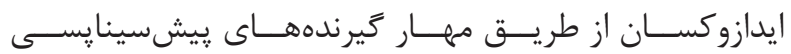

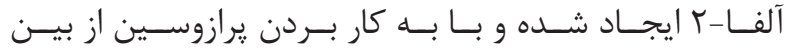

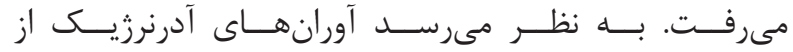

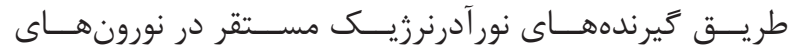

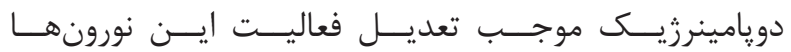

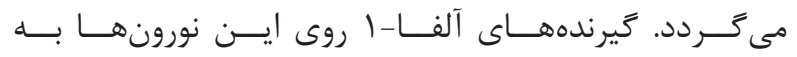

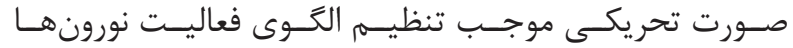

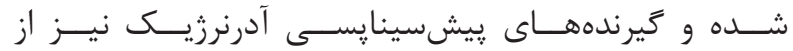

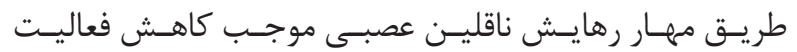

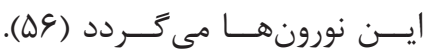

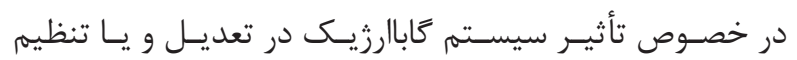

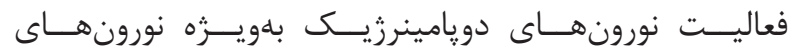

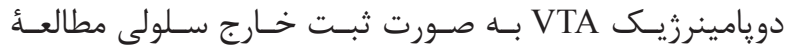

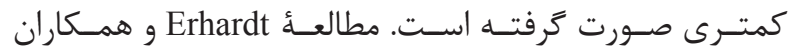

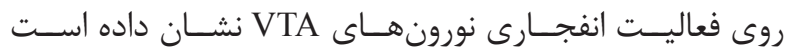

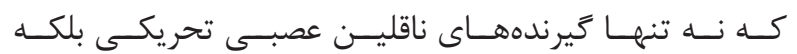

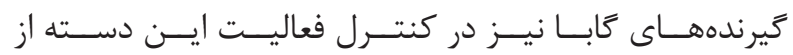

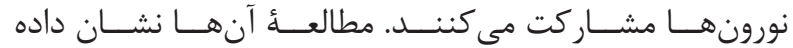

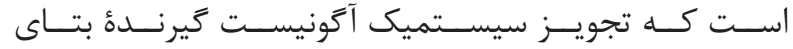

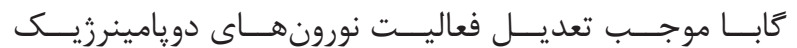

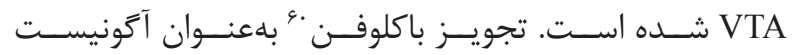

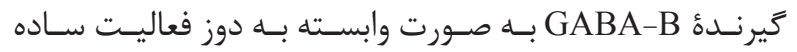

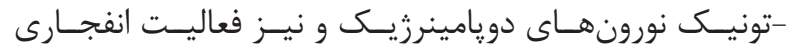

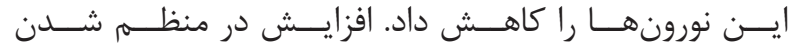

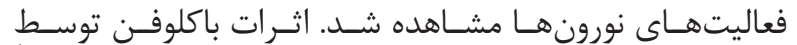

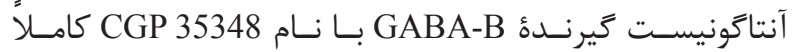

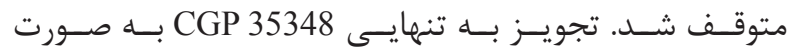

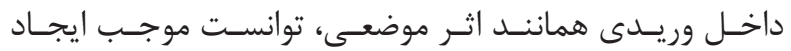

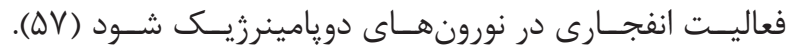

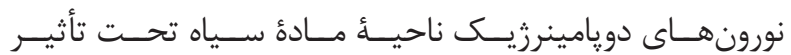

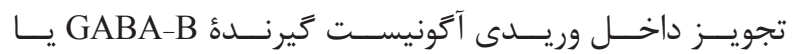

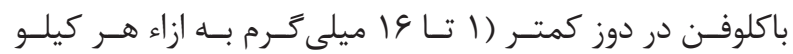

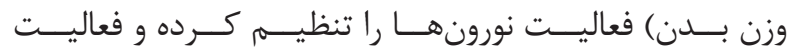

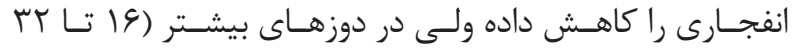

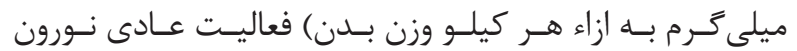

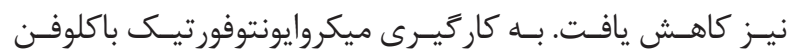

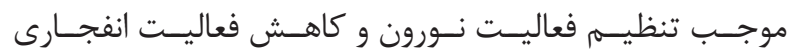

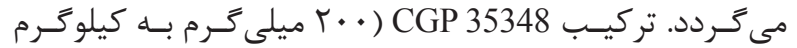

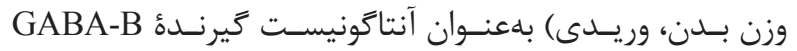

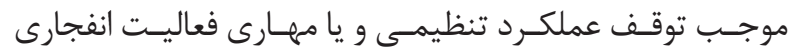

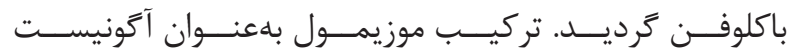

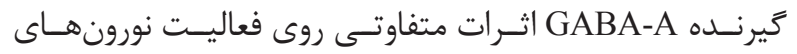

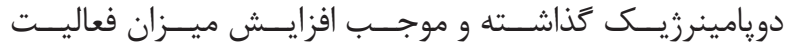

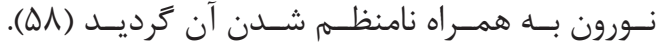

${ }^{56}$ Monophasically

57 Prazosin

${ }^{58}$ Idazoxan

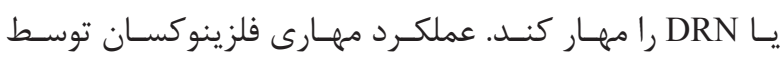

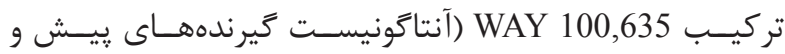

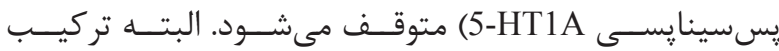

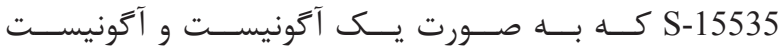

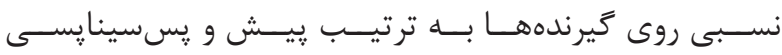

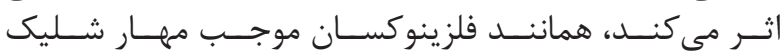

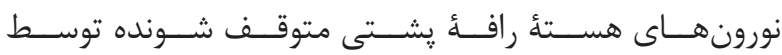

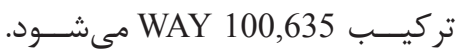

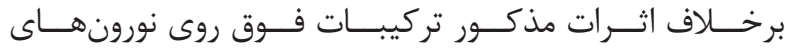

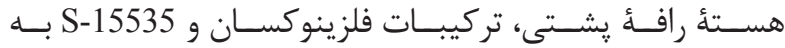

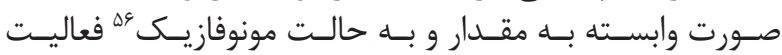

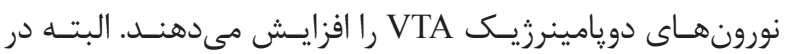

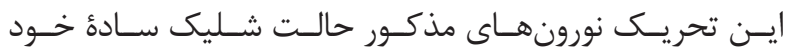

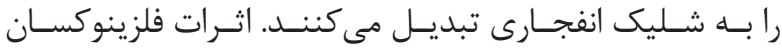

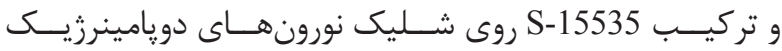

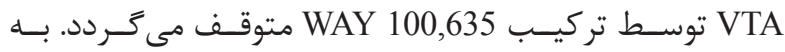

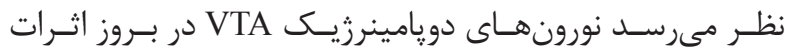

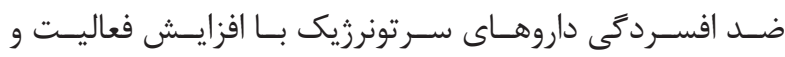

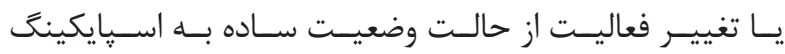

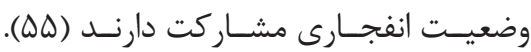

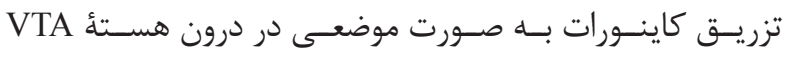

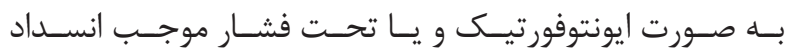

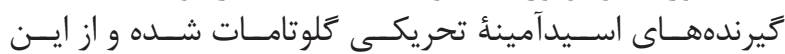

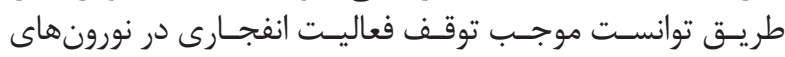

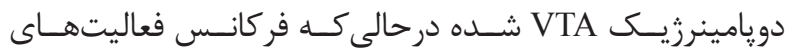

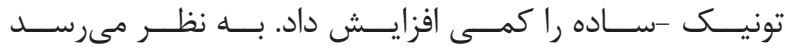

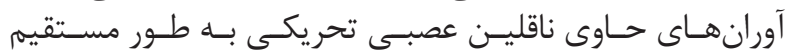

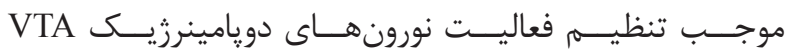

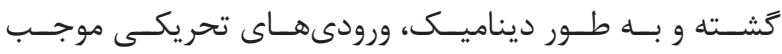

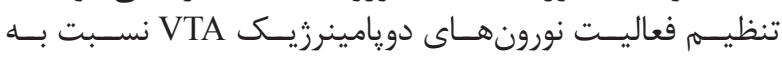

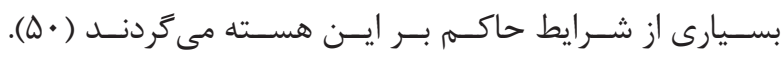

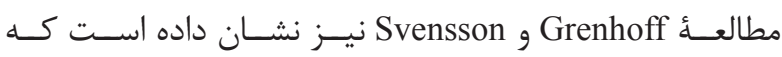

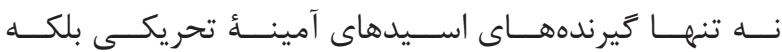

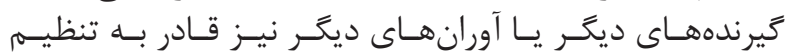

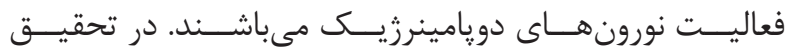

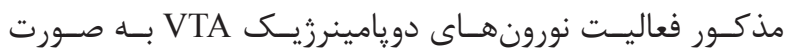

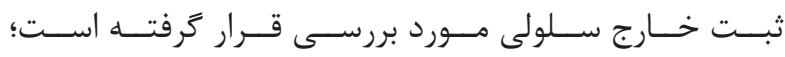

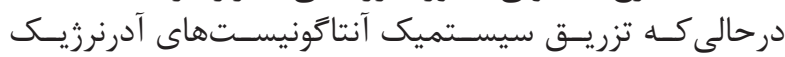

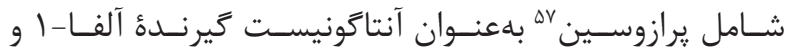

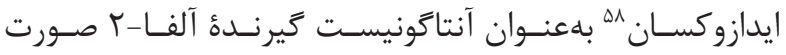

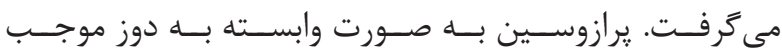

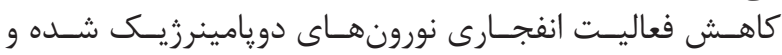

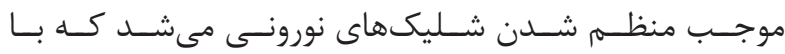

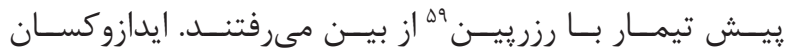

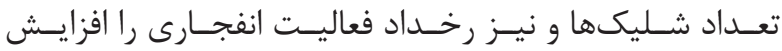

${ }^{59}$ Reserpine

${ }^{60}$ Baclofen 


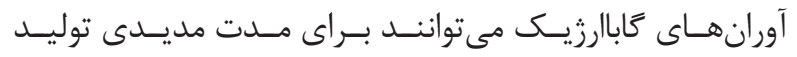

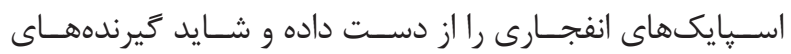

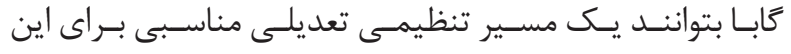

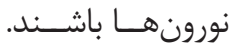

$$
\text { تشكر و قدردانى }
$$

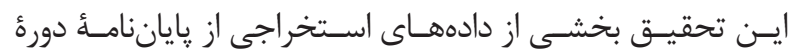

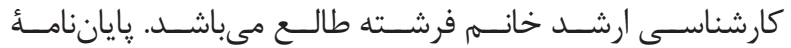

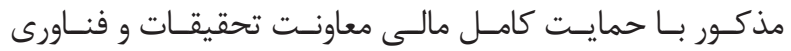

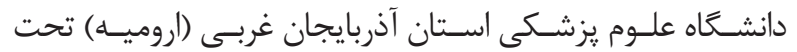

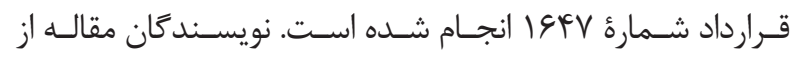

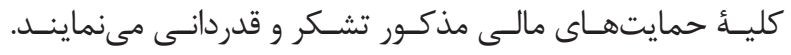

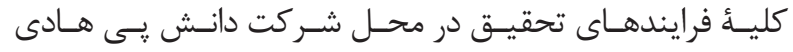

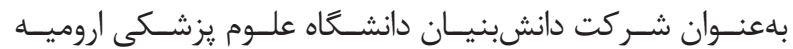

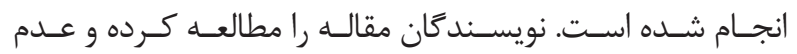

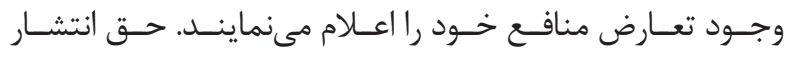

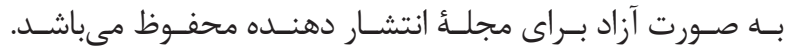

1. Anakwenze CP, Coronado-Interis E, Aung M, Jolly PE. A theory-based intervention to improve breast cancer awareness and screening in jamaica. prevention science: the official journal of the society for prevention research. 2015; 16(4): 578-85.

2. Luscher C, Malenka RC. Drug-evoked synaptic plasticity in addiction: from molecular changes to circuit remodeling. Neuron. 2011; 69(4): 650-63.

3. Nestler EJ, Carlezon WA, Jr. The mesolimbic dopamine reward circuit in depression. Biological psychiatry. Biol Psychiatry. 2006; 59(12): 1151-9.

4. Wise RA. Dopamine, learning and motivation. Nature reviews Neuroscience. 2004; 5(6): 483-94.

5. Margolis EB, Lock H, Hjelmstad GO, Fields HL. The ventral tegmental area revisited: is there an electrophysiological marker for dopaminergic neurons? J Physiol. 2006; 577(Pt 3): 907-24.

6. Nair-Roberts RG, Chatelain-Badie SD, Benson E, White-Cooper H, Bolam JP, Ungless MA. Stereological estimates of dopaminergic, GABAergic and glutamatergic neurons in the ventral tegmental area, substantia nigra and retrorubral field in the rat. Neuroscience. 2008; 152(4): 1024-31.

7. Swanson LW. The projections of the ventral tegmental area and adjacent regions: a combined fluorescent retrograde tracer and immunofluorescence study in the rat. Brain Res Bull. 1982; 9(1-6): 321-53.

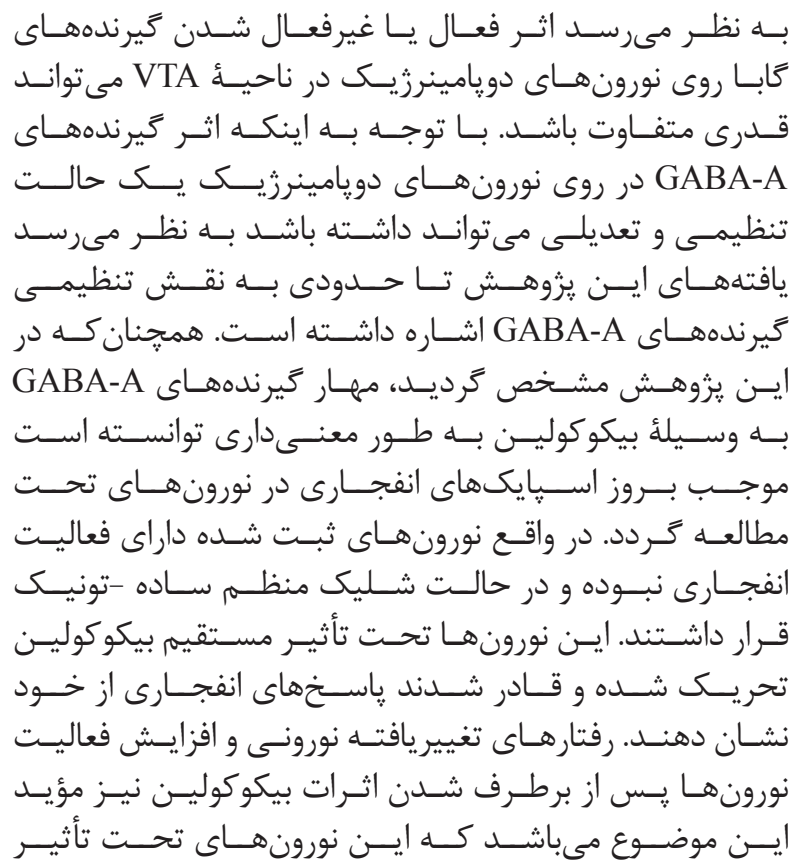

منابع

8. Yamaguchi T, Wang HL, Li X, Ng TH, Morales M. Mesocorticolimbic glutamatergic pathway. J Neurosci. 2011; 31(23): 8476-90.

9. Albanese A, Minciacchi D. Organization of the ascending projections from the ventral tegmental area: a multiple fluorescent retrograde tracer study in the rat. J Comp Neurol. 1983; 216(4): 406-20.

10. Brinschwitz K, Dittgen A, Madai VI, Lommel R, Geisler S, Veh RW. Glutamatergic axons from the lateral habenula mainly terminate on GABAergic neurons of the ventral midbrain. Neuroscience. 2010; 168(2): 463-76.

11. Jhou TC, Geisler S, Marinelli M, Degarmo BA, Zahm DS. The mesopontine rostromedial tegmental nucleus: A structure targeted by the lateral habenula that projects to the ventral tegmental area of Tsai and substantia nigra compacta. J Comp Neurol. 2009; 513(6): 566-96.

12. Tan KR, Yvon C, Turiault M, Mirzabekov JJ, Doehner J, Labouèbe G, et al. GABA neurons of the VTA drive conditioned place aversion. Neuron. 2012; 73(6): 1173-83.

13. van Zessen R, Phillips JL, Budygin EA, Stuber GD. Activation of VTA GABA neurons disrupts reward consumption. Neuron. 2012; 73(6): 1184-94.

14. Beier KT, Steinberg EE, DeLoach KE, Xie S, Miyamichi K, Schwarz L, et al. Circuit architecture of 
VTA dopamine neurons revealed by systematic inputoutput mapping. Cell. 2015; 162(3): 622-34.

15. Kudo T, Uchigashima M, Miyazaki T, Konno K, Yamasaki M, Yanagawa Y, et al. Three types of neurochemical projection from the bed nucleus of the stria terminalis to the ventral tegmental area in adult mice. J Neurosci. 2012; 32(50): 18035-46.

16. Zahm DS, Cheng AY, Lee TJ, Ghobadi CW, Schwartz $\mathrm{ZM}$, Geisler S, et al. Inputs to the midbrain dopaminergic complex in the rat, with emphasis on extended amygdalarecipient sectors. J Comp Neurol. 2011; 519(16): 3159-88.

17. Kaufling J, Waltisperger E, Bourdy R, Valera A, Veinante P, Freund-Mercier MJ, et al. Pharmacological recruitment of the GABAergic tail of the ventral tegmental area by acute drug exposure. Br J Pharmacol. 2010; 161(8): 1677-91.

18. Schultz W. Dopamine neurons and their role in reward mechanisms. Curr Opin Neurobiol. 1997; 7(2): 191-7.

19. Schultz W, Dayan P, Montague PR. A neural substrate of prediction and reward. Science. 1997; 275(5306): 1593-9.

20. Schultz W. Predictive reward signal of dopamine neurons. J Neurophysiol. 1998; 80(1): 1-27.

21. Cooper DC. The significance of action potential bursting in the brain reward circuit. Neurochem Int. 2002; 41(5): 333-40.

22. Komendantov AO, Komendantova OG, Johnson SW, Canavier CC. A modeling study suggests complementary roles for GABAA and NMDA receptors and the SK channel in regulating the firing pattern in midbrain dopamine neurons. J Neurophysiol. 2004; 91(1): 346-57.

23. Grace AA. The tonic/phasic model of dopamine system regulation and its implications for understanding alcohol and psychostimulant craving. Addiction. 2000; 95(2): 119-28.

24. Schultz W. Getting formal with dopamine and reward. Neuron. 2002; 36(2): 241-63.

25. GraceAA, Floresco SB, Goto Y, Lodge DJ. Regulation of firing of dopaminergic neurons and control of goaldirected behaviors. Trends Neurosci. 2007; 30(5): 220-7.

26. Melis M, Pillolla G, Perra S, Colombo G, Muntoni AL, Pistis M. Electrophysiological properties of dopamine neurons in the ventral tegmental area of
Sardinian alcohol-preferring rats. Psychopharmacology (Berl). 2009; 201(4): 471-81.

27. Heikkinen AE, Moykkynen TP, Korpi ER. Longlasting modulation of glutamatergic transmission in VTA dopamine neurons after a single dose of benzodiazepine agonists. Neuropsychopharmacology. 2009; 34(2): 290-8.

28. Saal D, Dong Y, Bonci A, Malenka RC. Drugs of abuse and stress trigger a common synaptic adaptation in dopamine neurons. Neuron. 2003; 37(4): 577-82.

29. Ikemoto S, Murphy JM, McBride WJ. Self-infusion of $\operatorname{GABA}(\mathrm{A})$ antagonists directly into the ventral tegmental area and adjacent regions. Behav Neurosci. 1997; 111(2): 369-80.

30. Ikemoto S, Murphy JM, McBride WJ. Regional differences within the rat ventral tegmental area for muscimol self-infusions. Pharmacol Biochem Behav. 1998; 61(1): 87-92.

31. Laviolette SR, van der Kooy D. GABA(A) receptors in the ventral tegmental area control bidirectional reward signalling between dopaminergic and nondopaminergic neural motivational systems. Eur $\mathrm{J}$ Neurosci. 2001; 13(5): 1009-15.

32. Kodangattil JN, Dacher M, Authement ME, Nugent FS. Spike timing-dependent plasticity at GABAergic synapses in the ventral tegmental area. J Physiol. 2013; 591(19): 4699-710.

33. Schweimer JV, Coullon GS, Betts JF, Burnet PW, Engle SJ, Brandon NJ, et al. Increased burst-firing of ventral tegmental area dopaminergic neurons in D-amino acid oxidase knockout mice in vivo. Eur $\mathrm{J}$ Neurosci. 2014; 40(7): 2999-3009.

34. Li W, Doyon WM, Dani JA. Quantitative unit classification of ventral tegmental area neurons in vivo. J Neurophysiol. 2012; 107(10): 2808-20.

35. Wang SS, Wei CL, Liu ZQ, Ren W. The influence of L-glutamate and carbachol on burst firing of dopaminergic neurons in ventral tegmental area. Sheng li xue bao: [Acta physiologica Sinica]. 2011; 63(1): 25-30.

36. Maggi CA, Meli A. Suitability of urethane anesthesia for physiopharmacological investigations in various systems Part 1: General considerations. Experientia. 1986; 42(2): 109-14.

37. Paxinos G, Watson C. The rat brain in stereotaxic coordinates. $6^{\text {th }}$ ed. San Diego: Academic Press. 2007. 


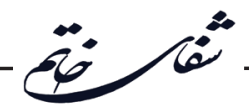

38. Pinault D. The Juxtacellular Recording-Labeling Technique. Neuromethods. 2010; 54: 41-75.

39. White FJ, Wang RY. Electrophysiological evidence for A10 dopamine autoreceptor subsensitivity following chronic D-amphetamine treatment. Brain Res. 1984; 309(2): 283-92.

40. Mansvelder HD, Keath JR, McGehee DS. Synaptic mechanisms underlie nicotine-induced excitability of brain reward areas. Neuron. 2002; 33(6): 905-19.

41. Marinelli M, Cooper DC, Baker LK, White FJ. Impulse activity of midbrain dopamine neurons modulates drug-seeking behavior. Psychopharmacology (Berl). 2003; 168(1-2): 84-98.

42. Ungless MA, Magill PJ, Bolam JP. Uniform inhibition of dopamine neurons in the ventral tegmental area by aversive stimuli. Science. 2004; 303(5666): 2040-2.

43. Hyland BI, Reynolds JNJ, Hay J, Perk CG, Miller R. Firing modes of midbrain dopamine cells in the freely moving rat. Neuroscience. 2002; 114(2): 475-92.

44. Shi WX. Slow oscillatory firing: a major firing pattern of dopamine neurons in the ventral tegmental area. J Neurophysiol. 2005; 94(5): 3516-22.

45. Grace AA, Bunney BS. The control of firing pattern in nigral dopamine neurons: single spike firing. J Neurosci. 1984; 4(11): 2866-76.

46. Miller JD, Sanghera MK, German DC. Mesencephalic dopaminergic unit activity in the behaviorally conditioned rat. Life Sci. 1981;29(12): 1255-63.

47. Freeman AS, Bunney BS. Activity of A9 and A10 dopaminergic neurons in unrestrained rats: further characterization and effects of apomorphine and cholecystokinin. Brain Res. 1987; 405(1): 46-55.

48. Grenhoff J, Ugedo L, Svensson TH. Firing patterns of midbrain dopamine neurons: differences between A9 and A10 cells. Acta Physiol Scand. 1988; 134(1): 127-32.

49. Steinfels GF, Heym J, Jacobs BL. Single unit activity of dopaminergic neurons in freely moving cuts. Life Sci. 1981; 29(14): 1435-42.

50. Charlety PJ, Grenhoff J, Chergui K, De la Chapelle
B, Buda M, Svensson TH, et al. Burst firing of mesencephalic dopamine neurons is inhibited by somatodendritic application of kynurenate. Acta Physiol Scand. 1991; 142(1): 105-12.

51. Erhardt S, Engberg G. Increased phasic activity of dopaminergic neurones in the rat ventral tegmental area following pharmacologically elevated levels of endogenous kynurenic acid. Acta Physiol Scand. 2002; 175(1): 45-53.

52. Linderholm KR, Alm MT, Larsson MK, Olsson SK, Goiny M, Hajos M, et al. Inhibition of kynurenine aminotransferase II reduces activity of midbrain dopamine neurons. Neuropharmacology. 2016; 102: 42-7.

53. Schwieler L, Engberg G, Erhardt S. Clozapine modulates midbrain dopamine neuron firing via interaction with the NMDA receptor complex. Synapse. 2004; 52(2): 114-22.

54. Schwieler L, Linderholm KR, Nilsson-Todd LK, Erhardt S, Engberg G. Clozapine interacts with the glycine site of the NMDA receptor: electrophysiological studies of dopamine neurons in the rat ventral tegmental area. Life Sci. 2008; 83(5-6): 170-5.

55. Lejeune F, Millan MJ. Induction of burst firing in ventral tegmental area dopaminergic neurons by activation of serotonin (5-HT)1A receptors: WAY 100,635-reversible actions of the highly selective ligands, flesinoxan and S 15535. Synapse. 1998; 30(2): 172-80.

56. Grenhoff J, Svensson TH. Prazosin modulates the firing pattern of dopamine neurons in rat ventral tegmental area. Eur J Pharmacol. 1993; 233(1): 7984.

57. Erhardt S, Mathe JM, Chergui K, Engberg G, Svensson TH. GABA(B) receptor-mediated modulation of the firing pattern of ventral tegmental area dopamine neurons in vivo. Naunyn Schmiedebergs Arch Pharmacol. 2002; 365(3): 173-80.

58. Engberg G, Kling-Petersen $T$, Nissbrandt $H$. GABAB-receptor activation alters the firing pattern of dopamine neurons in the rat substantia nigra. Synapse. 1993; 15(3): 229-38. 